\title{
NEEDLE MORPHO-ANATOMY AND POLLEN MORPHO- PHYSIOLOGY OF SELECTED CONIFERS IN URBAN CONDITIONS
}

\author{
NiKolić, M. B..$^{1 *}$-Stefanović, A. M. ${ }^{2}$ - Veselinović, M. M. ${ }^{1}$ - Milanović, D. S. ${ }^{3,4}-$ \\ Mladenović, D. K. ${ }^{1}$ - Mitrović, Ž. S. ${ }^{1}$ - EREMIJA, M. S. ${ }^{1}$ - RAKONJAC, B. LJ. ${ }^{1}$ \\ ${ }^{1}$ Institute of Forestry, Kneza Višeslava 3, 11000 Belgrade, Serbia \\ (e-mails:smikitis@gmail.com; mvcetiri@gmail.com; katarina.mladenovic@gmail.com; \\ mitrovicsuzana79@gmail.com; sasaeremija@gmail.com; ljrakonjac@yahoo.com; phonelfax: \\ +381-11-254-5969) \\ ${ }^{2}$ Institute for Biological Research "Siniša Stanković", University of Belgrade \\ Boulevard Despota Stefana 142, 11060 Belgrade, Serbia \\ (e-mails: milenast983@gmail.com; phone: +381-11-207-8300; fax: +381-11-276-1433) \\ ${ }^{3}$ Faculty of Forestry, University of Belgrade, Kneza Višeslava 1, 11000 Belgrade, Serbia \\ (e-mail: slobodan.milanovic@sfb.bg.ac.rs; phone: +381-11-305-3882; fax: +381-11-254-5485) \\ ${ }^{4}$ Faculty of Forestry and Wood Technology, Mendel University in Brno \\ Zemédèlská 3, 61300 Brno, Czech Republic \\ (e-mail: slobodan.milanovic@mendelu.cz; phonelfax:+420-545-211-128) \\ *Corresponding author \\ e-mail:smikitis@gmail.com \\ (Received 26 $6^{\text {th }}$ Oct 2018; accepted $7^{\text {th }}$ Jan 2019)
}

\begin{abstract}
Comparison of twelve conifer species (Abies alba, A. concolor, A. nordmanniana, A. pinsapo, Cedrus atlantica, C. deodara, Picea abies, P. omorika, P. pungens, Pseudotsuga menziesii, Taxus baccata, and Pinus nigra) in the sense of needle morpho-anatomy and pollen morpho-physiology, in correlation to air pollution, was performed for the first time. Analyzed properties of species were also compared with literature sources. Listed conifers were investigated in five Belgrade parks, characterised by different degrees of air pollution, especially $\mathrm{CO}_{2}$. Their rank, I-V, was performed from non-polluted to heavily-polluted parks. Ranking in the sense of needle morpho-anatomy and pollen morpho-physiology did not match expected ones, but park V remained the worst for many analyzed species. Trees with shorter needles had greater stomatal density, which was particularly prominent in A. alba, A. nordmanniana, P. abies, P. omorika, P. nigra and T. baccata. The pollen grains of $C$. atlantica and $T$. baccata were the most sensitive to air pollution. In some analyzed species distance of particular trees close to the heavy traffic also was in correlation with needle dimensions ( $P$. omorika, A. concolor, A. nordmanniana, $P$. nigra), stomatal density (A. alba, P. abies, $P$. omorika, $P$. pungens) and pollen vitality (A. pinsapo, C. atlantica, P. menziessi, P. nigra, and T. baccata).
\end{abstract}

Keywords: cedar, fir, pine, spruce, yew

\section{Introduction}

The increasing content of air pollutants, such as $\mathrm{CO}_{2}, \mathrm{SO}_{2}$, nitrogen oxides, soot, dust, etc. is always present in urban habitats and produces an adverse impact on growth and health of the living world. Since plants absorb chemicals and deposit solid particles, green areas, such as parks, are essential for the quality of life in cities. In addition, plants suffer changes which lead to their slow development, to diminished resistance to disease and pest attacks and, ultimately, to their death. These changes could be visible 
(macro-changes) or less visible (micro-changes). Both of them are reliable indicators of the level of vulnerability of urban habitats.

Many scienticists tried to imitate polluted habitats and trees response to them. Fumigation of Picea omorika's pollen with $\mathrm{SO}_{2}$ resulted in reduced seed production (Krug, 1990). Fumigation of the needles of seedlings of Scots pine (Pinus sylvestris) and Norway spruce (Picea abies) with $\mathrm{SO}_{2}, \mathrm{O}_{3}$ and combination of these two gases greatly reduced soluble carbohydrates in the needles taken from $P$. sylvestris, but to a lesser content in case of needles taken from P. abies (Peace et al., 1995). Furthermore, when field with needles of 3-year-old seedlings of Scots pine was fumigated with relatively low concentration of $\mathrm{SO}_{2}$, their needles emitted $\mathrm{H}_{2} \mathrm{~S}$. This emission depended of light and $\mathrm{SO}_{2}$ concentration (Hällgren and Fredrikson, 1982). On the other hand, some investigations showed that conifer species (Austrian pine, cypress and larch) influenced the lowest level of $\mathrm{NO}_{2}, \mathrm{O}_{3}$ and $\mathrm{NH}_{3}$ in the air (Donovan et al., 2005). Conifers also delay ultra-fine dust particles more effectively than broadleaved trees (Freer-Smith et al., 2005). Furthermore, it should be noted that only evergreen and conifer species delay leaves and transpire throughout the whole year. Their transpiration is important especially in winter, when the level of air pollutants is extremely high, or following heavy air pollution (in the period of defoliation of deciduous trees). There are four main ways that urban trees affect air quality: (1) temperature reduction and other microclimatic effects, (2) removal of air pollutants, (3) emission of volatile organic compounds and tree maintenance emissions, and (4) energy effects on buildings (Nowak, 2002).

The purpose of the study is to evaluate, for the first time, the degree of vulnerability of twelve conifer species from five parks to air pollution by means of investigation of both morpho-anatomic leaf properties and morpho-physiological pollen traits. Furthermore, the influence of distance of trees from traffic was also taken into account. Increased content of $\mathrm{CO}_{2}$ leads to a decrease of stomatal density (Lin et al., 2001) and number of stomata (Woodward and Bazzaz, 1988). In Pinus crassifolia (Qiang et al., 2003) their positive correlation $(\mathrm{r}=0.63)$ were found. In one-year old needles of $P$. douglasii, Apple et al. (2000) reported that $\mathrm{CO}_{2}$ and temperature did not influence stomatal density. They stated that effects could be more visible later (after three years) and genetic influence is very strong, so stomatal density depends on needle elongation.

\section{Materials and methods}

\section{Plant materials, selected parks and trees}

Five Belgrade parks in Serbia (Europe), exposed to different levels of air pollution, were selected in 2015 (Fig. 1). Listed parks were ranked from non-polluted to very heavily-polluted Popović et al. (2016).

For needle and pollen studies twelve and nine tree species were used (Tables 1 and 2, respectively).

\section{Processing morpho-anatomic properties of needles}

Well-developed one-year old leaves (needles) of twelve conifers were collected for laboratory testing: morpho-anatomic analyses of needles, where length, width, shape, dry mass and stomatal density were measured. For the purpose of morpho-anatomic 
analyses, needles all around a tree crown were collected (ca. 20 needles). Stomata rows from abaxial needle side were obtained by the 'Kolodium method' (Wolf, 1950).

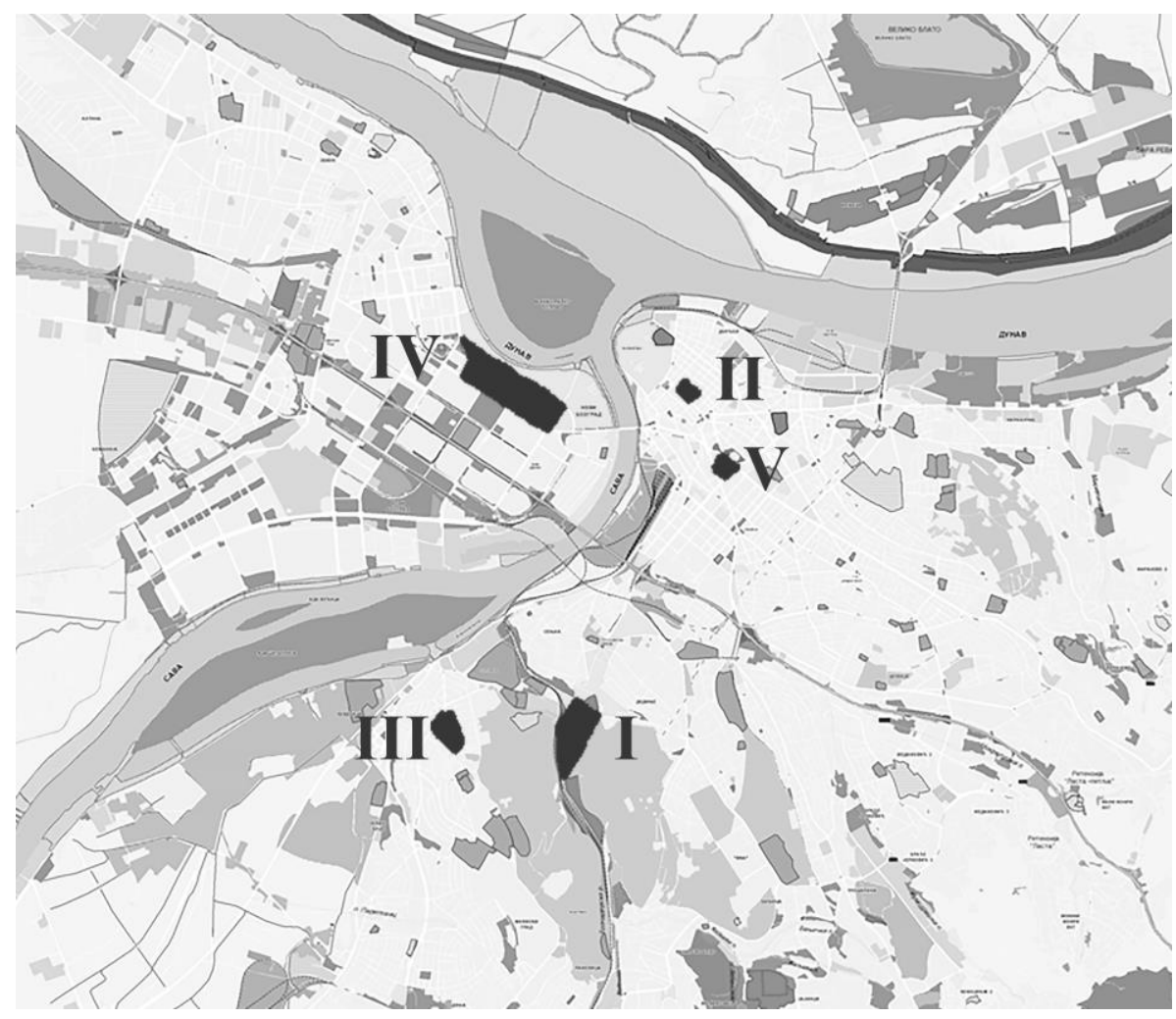

Figure 1. Shematic view of analysed Belgrade parks performed from non-polluted to heavilypolluted parks: I-Topčider; II-Academic; III-Banovo Brdo; IV-palace 'Serbia' and V-

Pioneer's park

Table 1. Number of trees per species sampled in five parks for needle studies

\begin{tabular}{|c|c|c|c|c|c|c|}
\hline \multirow{2}{*}{ No. } & \multirow{2}{*}{ Tree species } & \multicolumn{5}{|c|}{ Park No. } \\
\hline & & $\mathbf{I}$ & II & III & IV & $\mathbf{V}$ \\
\hline 1. & Abies alba Mill. & & & 1 & & 2 \\
\hline 2. & Abies concolor (Gordon) Lind. ex Hildebr. & & & 3 & & 2 \\
\hline 3. & Abies nordmanniana (Steven) Spach, 1841 & 3 & & & & \\
\hline 4. & Abies pinsapo Boiss. & & & & 1 & \\
\hline 5. & Cedrus atlantica (Endl.) Mann. ex Carrière & & 3 & 3 & 4 & 4 \\
\hline 6. & Cedrus deodara (Roxb.) G. Don) & & 1 & & & \\
\hline 7. & Picea abies (L.) Karst. & & & 1 & & 2 \\
\hline 8. & Picea omorika (Panč.) Pürkyné & 1 & & 2 & 2 & \\
\hline 9. & Picea pungens Engelm. & & & & 2 & 2 \\
\hline 10. & Pinus nigra J. F. Arnold & 3 & & 3 & 2 & 2 \\
\hline 11. & Pseudotsuga menziesii (Mirb.) Franco & & & 3 & 4 & 2 \\
\hline \multirow[t]{3}{*}{12.} & Taxus baccata $\mathrm{L}$. & & & & 3 & 2 \\
\hline & Sum & 7 & 4 & 16 & 18 & 18 \\
\hline & \multicolumn{5}{|l|}{ Sum } & 63 \\
\hline
\end{tabular}


Table 2. Number of trees per species sampled in five parks for pollen studies

\begin{tabular}{c|c|c|c|c|c|c}
\hline \multirow{2}{*}{ No. } & Tree species & \multicolumn{5}{|c}{ Park No. } \\
\cline { 3 - 6 } & Abies alba Mill. & I & II & III & IV & V \\
\hline 1. & & & 1 & & \\
2. & Abies nordmanniana (Steven) Spach, 1841 & 2 & & & & \\
3. & Abies pinsapo Boiss. & & & & 3 & \\
4. & Cedrus atlantica (Endl.) Mann. ex Carrière & & 1 & 3 & 3 & 2 \\
5. & Picea abies (L.) Karst. & & 1 & & \\
6. & Picea omorika (Panč.) Pürkyné & & & 2 & 3 & \\
7. & Pinus nigra J. F. Arnold & & & 3 & 3 & 3 \\
8. & Pseudotsuga menziesii (Mirb.) Franco & & & 3 & & 1 \\
9. & Taxus baccata L. & $\mathbf{2}$ & $\mathbf{1}$ & $\mathbf{1 5}$ & $\mathbf{1 5}$ & $\mathbf{8}$ \\
\hline & Sum & & & $\mathbf{4 1}$ \\
\hline
\end{tabular}

\section{Processing morpho-physiological properties of pollen grains}

Pollen was extracted from ripe strobili from nine trees in parks or extracted in laboratory after immersing twigs with half-ripe strobili in water. Observations of needle stomatal density and morpho-physiological analysis of pollen were performed using a light microscope (Leica Galen III) and included measurements of length and width of pollen grains, as well as their germinability and length of pollen tubes at $10 \%$ of sucrose. Measurements were carried out on 50 pollen grains per tree. The needle and pollen shape coefficient were calculated by the formula: $100 *$ width/length.

\section{Statistical analyses}

Mean values and differences between parks (at 95\% level), as well as analysis of variance were calculated in Statgraphics Centurion XVI, Version 16.1.11. Coefficient of correlation ( $r$ ) by linear regression analysis for all measured properties was performed.

\section{Results}

\section{Morpho-anatomic properties of needles}

Ranking of parks in Tables 3 and 4 were performed from heavily-polluted (V, IV) to moderate polluted (III, II) and non-polluted areas (I). The needles of A. alba, A. concolor, $P$. abies and $P$. pungens were significantly shorter in the park V (Table 3). Consequently, trees with shorter needles had greater stomatal density, which is particularly prominent in A. alba, A. nordmanniana, $P$. abies, $P$. omorika, $P$. nigra and T. baccata (Table 3; Fig. 2). Needles of P. omorika and P. nigra were significantly larger in the park IV (Table 3; Figs. 3 and 4). The needles of Taxus baccata, predominantly collected in the proximity of traffic crossroads in the park IV were shorter than in other parks (Fig. 4). Interestingly, the needles of Pseudotsuga menziesii were larger in the park V than in the park III (Table 3). Larger needles very often had a larger leaf-area, perimeter and dry mass. As presented in Table 3, especially with respect to morphology of needles, the parks are ranked as follows (from the best to the 
worst results): IV, II V, III and I. This ranking does not correlate to the ranking in terms of park pollution (I-V, from the best to the worst results). As a consequence, we could not conclude that morphological parameters of conifers are reliable indicators of air pollution. However, some species, such as $P$. omorika, $P$. pungens, $P$. nigra and $T$. baccata had larger needles when situated deep inside the park (far from the heavy traffic). Furthermore, A. alba and $P$. abies also produced better results in parks with moderately polluted air than in heavily-polluted parks. With respect to stomatal density, the greatest differences between parks were observed in case of A. alba, A. concolor, $C$. atlantica and $T$. baccata. Many species had the greatest stomatal density in the most polluted parks (V and IV), but we could not generally conclude that stomatal density of conifers is a dependable indicator of air pollution.
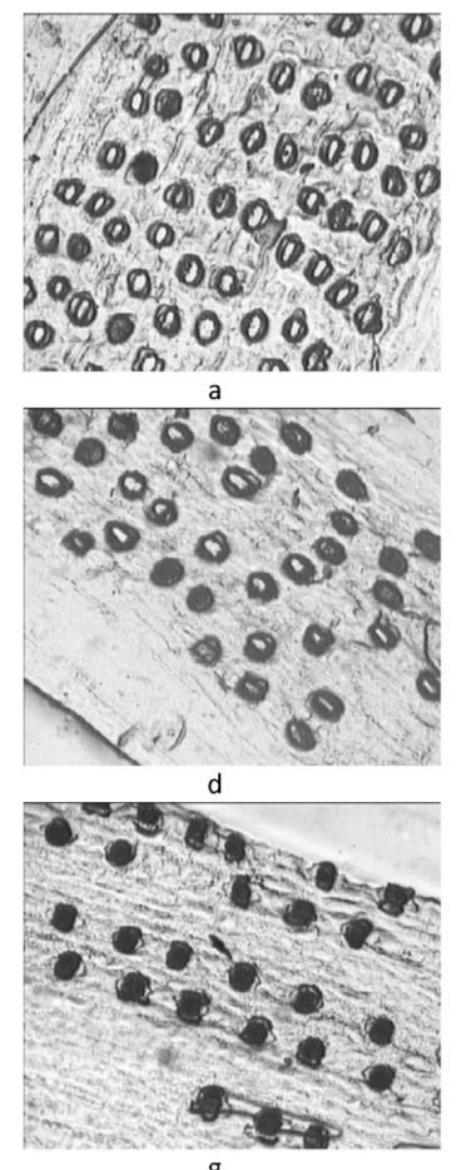

$\mathrm{g}$

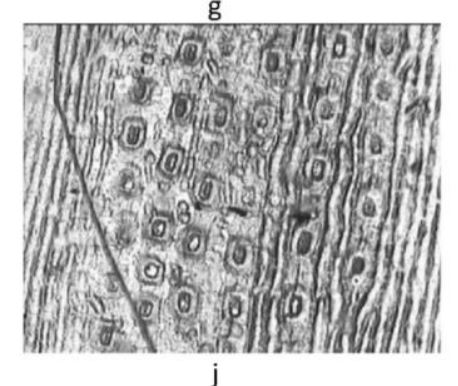

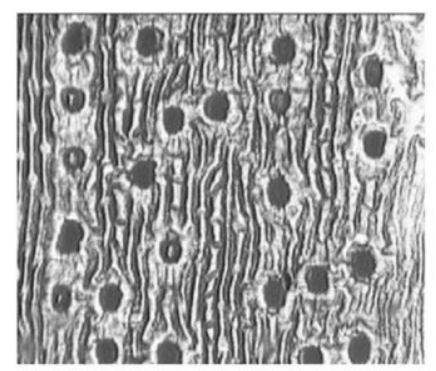

b

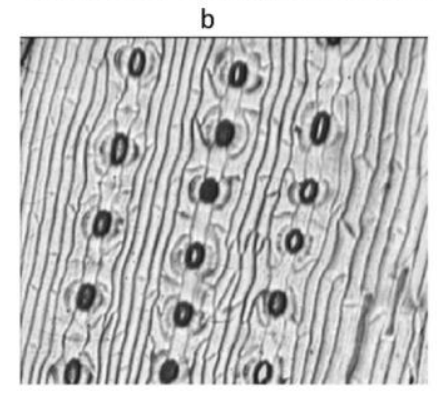

e
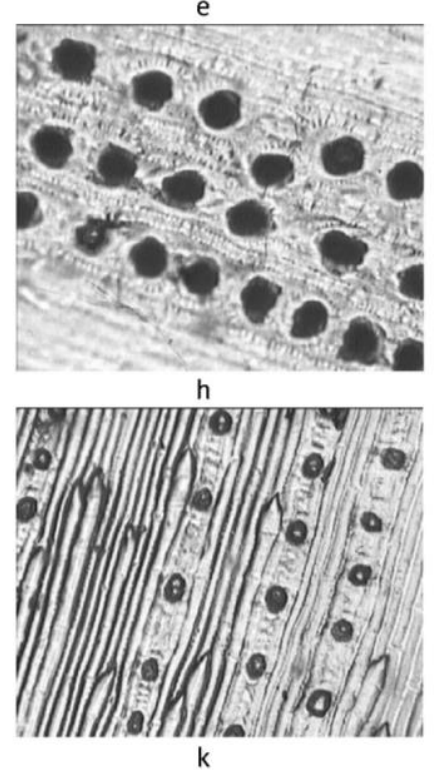

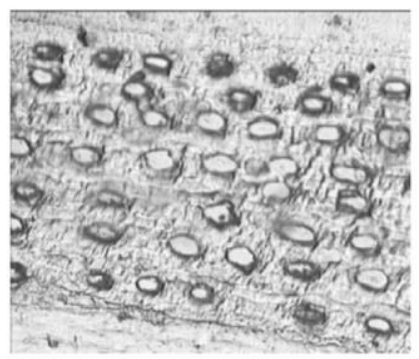

C
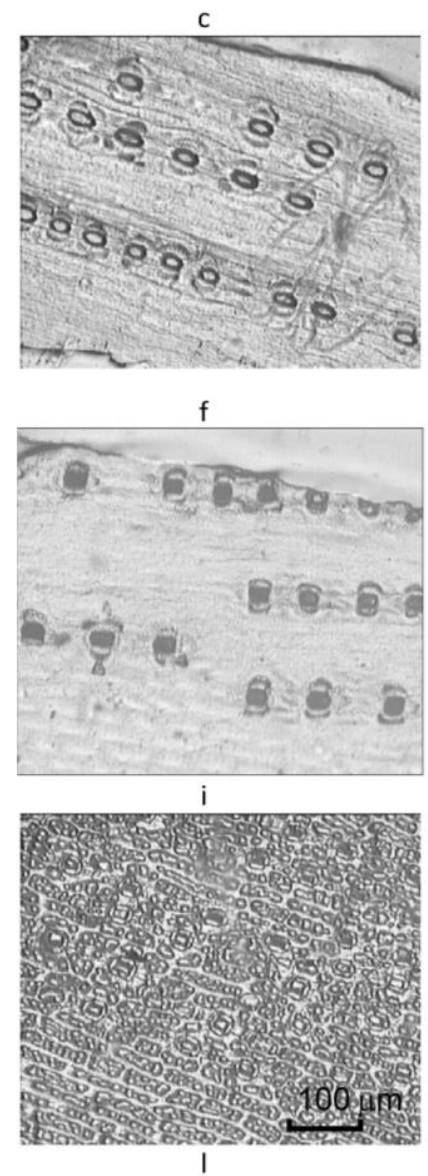

Figure 2. Rows of stomata on the abaxial needle side (objective 10x): Abies alba - V (a), A. concolor - III (b), A. nordmanniana - I (c), A. pinsapo - IV (d), Cedrus atlantica - III (e), C. deodara - II (f), Picea abies - V $(g)$, P. omorika - IV (h), P. pungens - IV (i), Pseudotsuga douglasii - III (j), Pinus nigra - III ( $k)$, Taxus baccata $-V(l)$ 


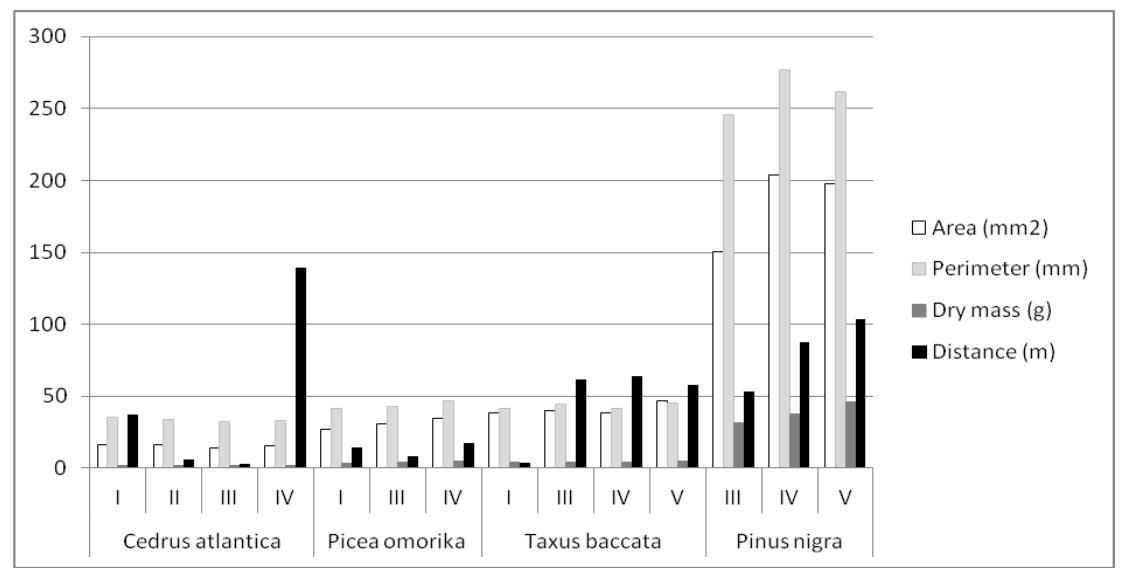

Figure 3. Graphical illustration of average values of needle area, perimeter, dry mass and distance from traffic in Cedrus atlantica, Picea omorika, Taxus baccata, and Pinus nigra

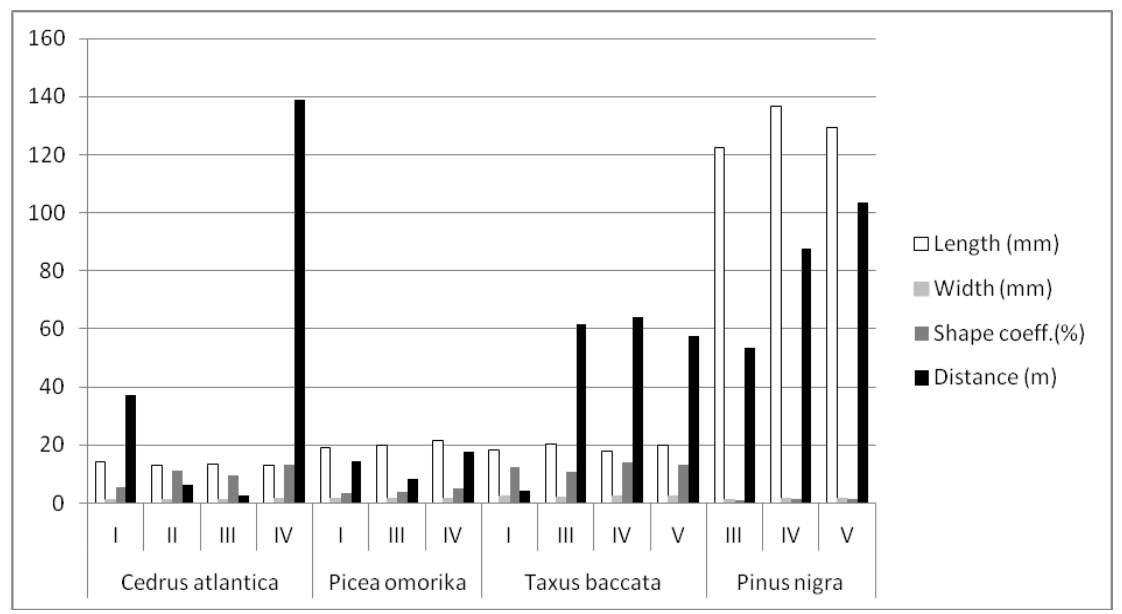

Figure 4. Graphical illustration of needle length, width, shape and distance from traffic in Cedrus atlantica, Picea omorika, Taxus baccata, and Pinus nigra

Table 3. Results of the morphological analyses of conifer leaves (needles), dry mass, stomatal density and distance of trees from traffic

\begin{tabular}{|c|c|c|c|c|c|c|c|c|c|}
\hline \multirow{3}{*}{$\begin{array}{c}\text { Park } \\
\text { No. }\end{array}$} & \multirow[b]{3}{*}{ Tree No. } & \multicolumn{5}{|c|}{ Needle morphology } & \multirow{3}{*}{$\begin{array}{c}\text { Dry } \\
\text { mass } \\
(\mathrm{g})\end{array}$} & \multirow{3}{*}{$\begin{array}{c}\begin{array}{c}\text { Stomatal } \\
\text { density } \\
\text { (number/ }\end{array} \\
\mathbf{m m}^{2} \text { ) }\end{array}$} & \multirow{3}{*}{$\begin{array}{c}\text { Distance } \\
\text { of trees } \\
\text { from } \\
\text { traffic } \\
(\mathbf{m})\end{array}$} \\
\hline & & \multirow[b]{2}{*}{$\begin{array}{c}\text { Area } \\
\left(\mathbf{m m}^{2}\right)\end{array}$} & \multirow[b]{2}{*}{$\begin{array}{l}\text { Perimeter } \\
(\mathbf{m m})\end{array}$} & \multicolumn{3}{|c|}{ Dimensions } & & & \\
\hline & & & & $\begin{array}{l}\text { Length } \\
(\mathbf{m m})\end{array}$ & $\begin{array}{l}\text { Width } \\
(\mathbf{m m})\end{array}$ & $\begin{array}{c}\text { Shape } \\
\text { coefficient } \\
(\%)\end{array}$ & & & \\
\hline \multirow{4}{*}{ V } & \multicolumn{9}{|c|}{ Abies alba } \\
\hline & 197 & 31.66 & 39.82 & 17.99 & 1.92 & 11.00 & 3.80 & 134.38 & 21.7 \\
\hline & 199 & 33.34 & 39.35 & 17.56 & 2.11 & 12.52 & 4.20 & 132.82 & 21.6 \\
\hline & $\begin{array}{l}\text { Mean } \\
\pm S D\end{array}$ & $\begin{array}{l}32.50 \\
\pm 1.19 \\
\end{array}$ & $\begin{array}{l}39.58 \\
\pm 0.33 \\
\end{array}$ & $\begin{array}{l}17.78 \\
\pm 0.30 \\
\end{array}$ & $\begin{array}{l}2.02 * \\
\pm 0.13\end{array}$ & $\begin{array}{l}11.76^{*} \\
\pm 1.07 \\
\end{array}$ & $\begin{array}{c}4.00 \\
\pm 0.28\end{array}$ & $\begin{array}{l}133.60 \\
\pm 1.10 \\
\end{array}$ & $\begin{array}{c}21.7 \\
\pm 0.07 \\
\end{array}$ \\
\hline \multirow[b]{2}{*}{ III } & 96 & 41.51 & 50.42 & 23.13 & 2.08 & 9.41 & 4.55 & 93.23 & 100.3 \\
\hline & $\begin{array}{c}\text { Mean } \\
\pm S D \\
\end{array}$ & $\begin{array}{l}41.51 \\
\pm 0.00 \\
\end{array}$ & $\begin{array}{c}50.42 \\
\pm 0.00 \\
\end{array}$ & $\begin{array}{l}23.13 \\
\pm 0.00 \\
\end{array}$ & $\begin{array}{c}2.08 \\
\pm 0.00 \\
\end{array}$ & $\begin{array}{c}9.41 \\
\pm 0.00 \\
\end{array}$ & $\begin{array}{r}4.55 \\
\pm 0.00 \\
\end{array}$ & $\begin{array}{l}93.23 \\
\pm 0.00 \\
\end{array}$ & $\begin{array}{l}100.3 \\
\pm 0.00 \\
\end{array}$ \\
\hline \multicolumn{2}{|c|}{$\begin{array}{l}\text { Mean } \\
\pm \text { SD }\end{array}$} & $\begin{array}{l}\text { 35.50* } \\
\pm \mathbf{5 . 2 7}\end{array}$ & $\begin{array}{l}43.20 * \\
\pm 6.26 \\
\end{array}$ & $\begin{array}{l}19.56^{*} \\
\pm 3.10 \\
\end{array}$ & $\begin{array}{c}2.04 \\
\pm 0.10 \\
\end{array}$ & $\begin{array}{c}10.98 \\
\pm 1.55 \\
\end{array}$ & \begin{tabular}{|c|}
4.18 \\
\pm 0.38 \\
\end{tabular} & $\begin{array}{l}120.14 * \\
\pm 23.32 \\
\end{array}$ & $\begin{array}{c}65.0 * \\
\pm 45.41 \\
\end{array}$ \\
\hline
\end{tabular}




\begin{tabular}{|c|c|c|c|c|c|c|c|c|c|}
\hline \multicolumn{10}{|c|}{ Abies concolor } \\
\hline \multirow{3}{*}{$\mathrm{V}$} & 220 & 77.18 & 84.39 & 39.90 & 2.30 & 6.08 & 14.30 & 68.91 & 24.4 \\
\hline & 221 & 82.11 & 96.35 & 46.14 & 2.03 & 4.68 & 12.85 & 94.61 & 20.8 \\
\hline & $\begin{array}{c}\text { Mean } \\
\pm S D\end{array}$ & $\begin{array}{l}79.65 \\
\pm 3.49 \\
\end{array}$ & $\begin{array}{l}90.37 \\
\pm 8.46 \\
\end{array}$ & $\begin{array}{l}43.02 \\
\pm 4.41 \\
\end{array}$ & $\begin{array}{l}2.17^{*} \\
\pm 0.19 \\
\end{array}$ & $\begin{array}{c}5.38 \\
\pm 0.99 \\
\end{array}$ & $\begin{array}{r}13.58 \\
\pm 1.02 \\
\end{array}$ & $\begin{array}{r}81.76 \\
\pm 18.17 \\
\end{array}$ & $\begin{array}{c}22.6 \\
\pm 2.54 \\
\end{array}$ \\
\hline \multirow{4}{*}{ III } & 248 & 106.70 & 98.07 & 46.20 & 2.83 & 6.19 & 21.33 & 84.58 & 19.6 \\
\hline & 282 & 65.69 & 76.59 & 36.18 & 2.11 & 6.06 & 8.80 & 88.23 & 26.2 \\
\hline & 283 & 89.22 & 95.01 & 45.26 & 2.25 & 5.28 & 13.70 & 93.51 & 22.8 \\
\hline & $\begin{array}{c}\text { Mean } \\
\pm S D\end{array}$ & $\begin{array}{l}87.40^{*} \\
\pm 20.58 \\
\end{array}$ & $\begin{array}{l}90.40^{*} \\
\pm 11.62 \\
\end{array}$ & $\begin{array}{l}42.82 * \\
\pm 5.53 \\
\end{array}$ & $\begin{array}{l}2.39^{*} \\
\pm 0.38\end{array}$ & $\begin{array}{c}5.78 \\
\pm 0.49 \\
\end{array}$ & $\begin{array}{c}14.61 \\
\pm 6.31 \\
\end{array}$ & $\begin{array}{l}88.77 \\
\pm 4.49 \\
\end{array}$ & $\begin{array}{c}22.8 \\
\pm 3.30 \\
\end{array}$ \\
\hline \multicolumn{2}{|c|}{$\begin{array}{l}\text { Mean } \\
\pm \text { SD }\end{array}$} & $\begin{array}{c}83.96 * \\
\pm 15.23\end{array}$ & $\begin{array}{l}90.39 \\
\pm 9.24\end{array}$ & $\begin{array}{c}42.91 \\
\pm 4.50\end{array}$ & $\begin{array}{l}2.29 * \\
\pm 0.31\end{array}$ & $\begin{array}{c}5.61 \\
\pm 0.66 \\
\end{array}$ & $\begin{array}{r}14.20 \\
\pm 4.53 \\
\end{array}$ & $\begin{array}{c}85.97 \\
\pm 10.36\end{array}$ & $\begin{array}{c}22.8 \\
\pm 2.66 \\
\end{array}$ \\
\hline \multicolumn{10}{|c|}{ Abies nordmanniana } \\
\hline \multirow{4}{*}{ I } & 149 & 31.96 & 40.50 & 18.41 & 1.84 & 10.32 & 3.87 & 116.25 & 74.1 \\
\hline & 150 & 28.52 & 38.34 & 17.33 & 1.84 & 10.66 & 3.95 & 184.22 & 70.3 \\
\hline & 153 & 22.98 & 31.31 & 13.83 & 1.82 & 13.23 & 2.40 & 127.97 & 59.7 \\
\hline & $\begin{array}{l}\text { Mean } \\
\pm S D\end{array}$ & $\begin{array}{l}28.44^{*} \\
\pm 4.53\end{array}$ & $\begin{array}{l}37.50^{*} \\
\pm 4.80\end{array}$ & $\begin{array}{l}16.91 * \\
\pm 2.39\end{array}$ & $\begin{array}{c}1.84 \\
\pm 0.01\end{array}$ & $\begin{array}{l}11.12 * \\
\pm 1.59\end{array}$ & $\begin{array}{c}3.41 \\
\pm 0.87\end{array}$ & $\begin{array}{l}142.81 \\
\pm 36.33\end{array}$ & $\begin{array}{c}68.0 \\
\pm 7.46\end{array}$ \\
\hline \multicolumn{2}{|c|}{$\begin{array}{c}\text { Mean } \\
\pm \text { SD }\end{array}$} & $\begin{array}{r}28.44 \\
\pm 4.53 \\
\end{array}$ & $\begin{array}{c}37.50 \\
\pm 4.80 \\
\end{array}$ & $\begin{array}{c}16.91 \\
\pm 2.39 \\
\end{array}$ & $\begin{array}{c}1.84 \\
\pm 0.01 \\
\end{array}$ & $\begin{array}{r}11.12 \\
\pm 1.59 \\
\end{array}$ & $\begin{array}{c}3.41 \\
\pm 0.87 \\
\end{array}$ & $\begin{array}{r}142.81 \\
\pm 36.33 \\
\end{array}$ & $\begin{array}{c}68.0 \\
\pm 7.46 \\
\end{array}$ \\
\hline \multicolumn{10}{|c|}{ Abies pinsapo } \\
\hline \multirow[b]{2}{*}{ IV } & 8 & 40.07 & 47.14 & 21.34 & 2.23 & 10.86 & 5.60 & 133.99 & 25.6 \\
\hline & $\begin{array}{c}\text { Mean } \\
\pm S D\end{array}$ & $\begin{array}{l}40.07 \\
\pm 0.00 \\
\end{array}$ & $\begin{array}{l}47.14 \\
\pm 0.00 \\
\end{array}$ & $\begin{array}{l}21.34 \\
\pm 0.00 \\
\end{array}$ & $\begin{array}{c}2.23 \\
\pm 0.00 \\
\end{array}$ & $\begin{array}{l}10.86 \\
\pm 0.00\end{array}$ & $\begin{array}{c}5.60 \\
\pm 0.00\end{array}$ & $\begin{array}{l}133.99 \\
\pm 0.00\end{array}$ & $\begin{array}{c}25.6 \\
\pm 0.00\end{array}$ \\
\hline \multicolumn{2}{|c|}{$\begin{array}{l}\text { Mean } \\
\pm \text { SD }\end{array}$} & $\begin{array}{r}40.07 \\
\pm 0.00 \\
\end{array}$ & $\begin{array}{r}47.14 \\
\pm 0.00 \\
\end{array}$ & $\begin{array}{r}21.34 \\
\pm 0.00 \\
\end{array}$ & $\begin{array}{c}2.23 \\
\pm 0.00 \\
\end{array}$ & $\begin{array}{r}10.86 \\
\pm 0.00 \\
\end{array}$ & $\begin{array}{c}5.60 \\
\pm 0.00 \\
\end{array}$ & $\begin{array}{c}133.99 \\
\pm 0.00 \\
\end{array}$ & $\begin{array}{c}25.6 \\
\pm 0.00 \\
\end{array}$ \\
\hline \multicolumn{10}{|c|}{ Cedrus atlantica } \\
\hline \multirow{5}{*}{$\mathrm{V}$} & 26 & 13.96 & 28.70 & 11.07 & 1.29 & 11.88 & 1.80 & - & 77.7 \\
\hline & 80 & 19.54 & 45.76 & 19.15 & 1.11 & 5.77 & 1.80 & 78.20 & 44.3 \\
\hline & 176 & 16.62 & 37.56 & 15.51 & 1.13 & 9.28 & 1.95 & 83.13 & 7.7 \\
\hline & 181 & 12.25 & 23.85 & 8.73 & 1.50 & 17.32 & 1.40 & 81.67 & 7.0 \\
\hline & $\begin{array}{c}\text { Mean } \\
\pm S D\end{array}$ & $\begin{array}{l}16.03 * \\
\pm 3.19 \\
\end{array}$ & $\begin{array}{l}35.50^{*} \\
\pm 9.70 \\
\end{array}$ & $\begin{array}{l}14.42 * \\
\pm 4.64 \\
\end{array}$ & $\begin{array}{r}1.20 \\
\pm 0.18 \\
\end{array}$ & $\begin{array}{r}11.06^{*} \\
\pm 4.86 \\
\end{array}$ & $\begin{array}{c}1.74 \\
\pm 0.24 \\
\end{array}$ & $\begin{array}{r}81.00 \\
\pm 2.53 \\
\end{array}$ & $\begin{array}{c}34.2 \\
\pm 33.84 \\
\end{array}$ \\
\hline \multirow{5}{*}{ IV } & 59 & 18.66 & 43.59 & 17.67 & 1.16 & 7.03 & 2.13 & - & 148.2 \\
\hline & 68 & 13.35 & 25.77 & 9.72 & 1.61 & 19.22 & 2.07 & - & 161.9 \\
\hline & 83 & 15.60 & 29.37 & 11.48 & 1.57 & 15.77 & 2.25 & 78.75 & 133.1 \\
\hline & 445 & 15.43 & 33.76 & 13.11 & 1.31 & 10.50 & 1.95 & 56.15 & 113.2 \\
\hline & $\begin{array}{c}\text { Mean } \\
\pm S D\end{array}$ & $\begin{array}{l}15.73^{*} \\
\pm 2.19\end{array}$ & $\begin{array}{l}32.90^{*} \\
\pm 7.70 \\
\end{array}$ & $\begin{array}{l}12.90^{*} \\
\pm 3.41 \\
\end{array}$ & $\begin{array}{l}1.42 * \\
\pm 0.21 \\
\end{array}$ & $\begin{array}{l}13.13^{*} \\
\pm 5.42 \\
\end{array}$ & $\begin{array}{c}2.10 \\
\pm 0.12 \\
\end{array}$ & $\begin{array}{r}67.45 \\
\pm 15.98 \\
\end{array}$ & $\begin{array}{r}139.1 \\
\pm 20.89 \\
\end{array}$ \\
\hline \multirow{4}{*}{ III } & 322 & 18.74 & 39.52 & 15.52 & 1.33 & 9.05 & 2.25 & 56.33 & 10.8 \\
\hline & 329 & 10.53 & 27.09 & 10.61 & 1.17 & 11.13 & 1.00 & 77.11 & 32.2 \\
\hline & 335 & 13.71 & 31.38 & 12.72 & 1.13 & 9.54 & 1.65 & 73.28 & 37.9 \\
\hline & $\begin{array}{c}\text { Mean } \\
\pm S D\end{array}$ & $\begin{array}{l}15.08 * \\
\pm 4.14\end{array}$ & $\begin{array}{l}33.78^{*} \\
\pm 6.31 \\
\end{array}$ & $\begin{array}{l}13.42 * \\
\pm 2.46\end{array}$ & $\begin{array}{l}1.22 * \\
\pm 0.11 \\
\end{array}$ & $\begin{array}{c}9.67 \\
\pm 1.09 \\
\end{array}$ & $\begin{array}{r}1.63 \\
\pm 0.62 \\
\end{array}$ & $\begin{array}{r}68.91 \\
\pm 11.06 \\
\end{array}$ & $\begin{array}{r}29.7 \\
\pm 14.29 \\
\end{array}$ \\
\hline \multirow{4}{*}{ II } & 45 & 18.47 & 40.16 & 13.27 & 1.34 & 8.88 & 2.05 & 51.77 & 113.6 \\
\hline & 47 & 17.21 & 32.41 & 12.47 & 1.59 & 13.52 & 2.45 & - & 104.2 \\
\hline & 57 & 12.37 & 28.23 & 11.41 & 1.18 & 10.89 & 1.30 & 65.63 & 5.6 \\
\hline & $\begin{array}{c}\text { Mean } \\
\pm S D\end{array}$ & $\begin{array}{l}16.02 * \\
\pm 3.22 \\
\end{array}$ & $\begin{array}{l}33.60^{*} \\
\pm 6.05\end{array}$ & $\begin{array}{l}13.05^{*} \\
\pm 0.93\end{array}$ & $\begin{array}{l}1.37 * \\
\pm 0.21 \\
\end{array}$ & $\begin{array}{l}11.10^{*} \\
\pm 2.33\end{array}$ & $\begin{array}{r}1.93 \\
\pm 0.58 \\
\end{array}$ & $\begin{array}{l}58.70 \\
\pm 9.80 \\
\end{array}$ & $\begin{array}{c}73.3 \\
+59.82 \\
\end{array}$ \\
\hline \multicolumn{2}{|c|}{$\begin{array}{l}\text { Mean } \\
\pm \text { SD }\end{array}$} & $\begin{array}{c}15.71 \\
\pm 2.84\end{array}$ & $\begin{array}{r}33.72 \\
\pm 6.89\end{array}$ & $\begin{array}{r}13.31 \\
\pm 2.99\end{array}$ & $\begin{array}{l}1.32 * \\
\pm 0.18\end{array}$ & $\begin{array}{r}11.30 \\
\pm 3.84\end{array}$ & $\begin{array}{c}1.86 \\
\pm 0.40\end{array}$ & $\begin{array}{c}70.20 \\
+11.75\end{array}$ & $\begin{array}{c}69.0 \\
+56.93 \\
\end{array}$ \\
\hline
\end{tabular}




\begin{tabular}{|c|c|c|c|c|c|c|c|c|c|}
\hline \multicolumn{10}{|c|}{ Cedrus deodara } \\
\hline \multirow[b]{2}{*}{ II } & 91 & 19.46 & 39.71 & 15.82 & 1.34 & 8.90 & 2.67 & 128.13 & 18.6 \\
\hline & $\begin{array}{c}\text { Mean } \\
\pm S D\end{array}$ & $\begin{array}{l}19.46 \\
\pm 0.00\end{array}$ & $\begin{array}{l}39.71 \\
\pm 0.00\end{array}$ & $\begin{array}{l}15.82 \\
\pm 0.00 \\
\end{array}$ & $\begin{array}{c}1.34 \\
\pm 0.00 \\
\end{array}$ & $\begin{array}{r}8.90 \\
\pm 0.00 \\
\end{array}$ & $\begin{array}{c}2.67 \\
\pm 0.00 \\
\end{array}$ & $\begin{array}{l}128.13 \\
\pm 0.00\end{array}$ & $\begin{aligned} & 18.6 \\
& \pm 0.00 \\
&\end{aligned}$ \\
\hline \multicolumn{2}{|c|}{$\begin{array}{l}\text { Mean } \\
\pm \text { SD } \\
\end{array}$} & $\begin{array}{r}19.46 \\
\pm 0.00 \\
\end{array}$ & $\begin{array}{c}39.71 \\
\pm 0.00 \\
\end{array}$ & $\begin{array}{r}15.82 \\
\pm 0.00 \\
\end{array}$ & $\begin{array}{c}1.34 \\
\pm 0.00 \\
\end{array}$ & $\begin{array}{c}8.90 \\
\pm 0.00 \\
\end{array}$ & $\begin{array}{c}2.67 \\
\pm 0.00 \\
\end{array}$ & $\begin{array}{l}128.13 \\
\pm 0.00 \\
\end{array}$ & $\begin{array}{c}18.6 \\
\pm 0.00 \\
\end{array}$ \\
\hline \multicolumn{10}{|c|}{ Picea abies } \\
\hline \multirow{3}{*}{$\mathrm{V}$} & 15 & 34.26 & 44.45 & 20.41 & 1.82 & 9.02 & 2.75 & 74.38 & 36.8 \\
\hline & 18 & 21.80 & 37.92 & 17.67 & 1.29 & 7.60 & 2.45 & 85.86 & 44.0 \\
\hline & $\begin{array}{c}\text { Mean } \\
\pm S D\end{array}$ & $\begin{array}{l}28.03 * \\
\pm 8.81\end{array}$ & $\begin{array}{l}41.18 * \\
\pm 4.62\end{array}$ & $\begin{array}{l}19.04 * \\
\pm 1.94\end{array}$ & $\begin{array}{l}1.55 * \\
\pm 0.37\end{array}$ & $\begin{array}{l}8.31 * \\
\pm 1.00 \\
\end{array}$ & $\begin{array}{c}2.60 \\
\pm 0.21 \\
\end{array}$ & $\begin{array}{r}80.12 \\
\pm 4.52 \\
\end{array}$ & $\begin{array}{c}40.4 \\
+5.09 \\
\end{array}$ \\
\hline \multirow[b]{2}{*}{ III } & 382 & 28.00 & 45.30 & 17.88 & 1.60 & 8.99 & 3.45 & - & 62.1 \\
\hline & $\begin{array}{c}\text { Mean } \\
\pm S D \\
\end{array}$ & $\begin{array}{l}28.00 \\
\pm 0.00 \\
\end{array}$ & $\begin{array}{r}45.30 \\
\pm 0.00 \\
\end{array}$ & $\begin{array}{l}17.88 \\
\pm 0.00 \\
\end{array}$ & $\begin{aligned} & 1.60 \\
& \pm 0.00 \\
&\end{aligned}$ & $\begin{array}{c}8.99 \\
\pm 0.00 \\
\end{array}$ & $\begin{array}{c}3.45 \\
\pm 0.00 \\
\end{array}$ & - & $\begin{array}{c}62.1 \\
\pm 0.00 \\
\end{array}$ \\
\hline \multicolumn{2}{|c|}{$\begin{array}{l}\text { Mean } \\
\pm \text { SD }\end{array}$} & $\begin{array}{l}28.02 * \\
\pm 6.23\end{array}$ & $\begin{array}{l}42.56 \\
\pm 4.04 \\
\end{array}$ & $\begin{array}{l}18.65 * \\
\pm 1.52\end{array}$ & $\begin{array}{c}1.57 * \\
\pm 0.27\end{array}$ & $\begin{array}{c}8.54 \\
\pm 0.81 \\
\end{array}$ & $\begin{array}{c}2.88 \\
\pm 0.51 \\
\end{array}$ & $\begin{array}{r}80.12 \\
\pm 4.52 \\
\end{array}$ & $\begin{array}{c}51.3 \\
\pm 13.03 \\
\end{array}$ \\
\hline \multicolumn{10}{|c|}{ Picea omorika } \\
\hline \multirow{3}{*}{ IV } & 362 & 34.74 & 49.02 & 22.81 & 1.70 & 7.54 & 4.61 & 103.54 & 115.9 \\
\hline & 495 & 34.26 & 44.45 & 20.41 & 1.82 & 9.02 & 5.20 & 105.00 & 51.2 \\
\hline & $\begin{array}{c}\text { Mean } \\
\pm S D\end{array}$ & $\begin{array}{l}34.50 \\
\pm 0.34 \\
\end{array}$ & $\begin{array}{l}46.73 * \\
\pm 3.23 \\
\end{array}$ & $\begin{array}{l}21.61 * \\
\pm 1.70 \\
\end{array}$ & $\begin{array}{l}1.76^{*} \\
\pm 0.08 \\
\end{array}$ & $\begin{array}{l}8.28 * \\
\pm 1.05 \\
\end{array}$ & $\begin{array}{c}4.91 \\
\pm 0.42 \\
\end{array}$ & $\begin{array}{l}104.27 \\
\pm 1.03 \\
\end{array}$ & $\begin{array}{c}83.5 \\
\pm 45.11 \\
\end{array}$ \\
\hline \multirow{3}{*}{ III } & 1 & 33.30 & 47.44 & 22.14 & 1.58 & 7.27 & 4.15 & 123.59 & 9.3 \\
\hline & 4 & 27.59 & 38.26 & 17.46 & 1.67 & 9.82 & 3.50 & 108.28 & 19.7 \\
\hline & $\begin{array}{c}\text { Mean } \\
\pm S D\end{array}$ & $\begin{array}{l}30.45^{*} \\
\pm 4.04\end{array}$ & $\begin{array}{l}42.85 * \\
\pm 6.49\end{array}$ & $\begin{array}{l}19.80^{*} \\
\pm 3.31 \\
\end{array}$ & $\begin{array}{c}1.63 \\
\pm 0.06 \\
\end{array}$ & $\begin{array}{l}8.55^{*} \\
\pm 1.80 \\
\end{array}$ & $\begin{array}{c}3.83 \\
\pm 0.46 \\
\end{array}$ & $\begin{array}{r}115.94 \\
\pm 10.82\end{array}$ & $\begin{array}{r}14.5 \\
\pm 7.35 \\
\end{array}$ \\
\hline \multirow[b]{2}{*}{ I } & 338 & 26.82 & 41.38 & 19.13 & 1.56 & 8.35 & 3.60 & - & 17.8 \\
\hline & $\begin{array}{c}\text { Mean } \\
\pm S D\end{array}$ & $\begin{array}{c}26.82 \\
\pm 0.00\end{array}$ & $\begin{array}{l}41.38 \\
\pm 0.00\end{array}$ & $\begin{array}{l}19.13 \\
\pm 0.00\end{array}$ & $\begin{array}{c}1.56 \\
\pm 0.00 \\
\end{array}$ & $\begin{array}{c}8.35 \\
\pm 0.00 \\
\end{array}$ & $\begin{array}{c}3.60 \\
\pm 0.00 \\
\end{array}$ & - & $\begin{array}{c}17.8 \\
\pm 0.00 \\
\end{array}$ \\
\hline \multicolumn{2}{|c|}{$\begin{array}{l}\text { Mean } \\
\pm \text { SD }\end{array}$} & $\begin{array}{c}\text { 31.34* } \\
\pm 3.82 \\
\end{array}$ & $\begin{array}{c}44.11 * \\
\pm 4.39\end{array}$ & $\begin{array}{c}20.39 * \\
\pm 2.18 \\
\end{array}$ & $\begin{array}{c}1.67 * \\
\pm 0.10 \\
\end{array}$ & $\begin{array}{c}8.40 \\
\pm 1.05 \\
\end{array}$ & $\begin{array}{c}4.21 \\
\pm 0.71 \\
\end{array}$ & $\begin{array}{l}110.10 \\
\pm 9.21 \\
\end{array}$ & $\begin{array}{c}38.6 \\
\pm 43.48 \\
\end{array}$ \\
\hline \multicolumn{10}{|c|}{ Picea pungens } \\
\hline \multirow{3}{*}{$\mathrm{V}$} & 23 & 37.44 & 52.75 & 24.60 & 1.77 & 7.47 & 5.80 & 75.47 & 65.9 \\
\hline & 25 & 29.44 & 45.27 & 21.12 & 1.52 & 7.31 & 5.60 & 80.39 & 75.0 \\
\hline & $\begin{array}{c}\text { Mean } \\
\pm S D\end{array}$ & $\begin{array}{l}33.44 * \\
\pm 5.66 \\
\end{array}$ & $\begin{array}{l}49.01 * \\
\pm 5.29 \\
\end{array}$ & $\begin{array}{l}22.86^{*} \\
\pm 2.46 \\
\end{array}$ & $\begin{array}{l}1.65^{*} \\
\pm 0.18 \\
\end{array}$ & $\begin{array}{r}7.39 \\
\pm 0.11 \\
\end{array}$ & $\begin{array}{c}5.70 \\
\pm 0.14 \\
\end{array}$ & $\begin{array}{l}77.93 \\
\pm 3.48 \\
\end{array}$ & $\begin{array}{c}70.5 \\
\pm 6.43 \\
\end{array}$ \\
\hline \multirow{3}{*}{ IV } & 272 & 35.63 & 53.76 & 25.36 & 1.52 & 6.10 & 6.55 & 107.19 & 148.6 \\
\hline & 273 & 50.09 & 64.51 & 30.42 & 1.83 & 6.08 & 10.70 & 82.03 & 149.5 \\
\hline & $\begin{array}{c}\text { Mean } \\
\pm S D \\
\end{array}$ & $\begin{array}{l}42.86 * \\
\pm 10.22 \\
\end{array}$ & $\begin{array}{l}59.13 * \\
\pm 7.60 \\
\end{array}$ & $\begin{array}{l}27.89 * \\
\pm 3.58 \\
\end{array}$ & $\begin{array}{l}1.68 * \\
\pm 0.22 \\
\end{array}$ & $\begin{array}{c}6.09 \\
\pm 0.01 \\
\end{array}$ & $\begin{array}{c}8.63 \\
\pm 2.93 \\
\end{array}$ & $\begin{array}{c}94.61 \\
\pm 17.79 \\
\end{array}$ & $\begin{array}{c}149.0 \\
\pm 0.64 \\
\end{array}$ \\
\hline \multicolumn{2}{|c|}{$\begin{array}{l}\text { Mean } \\
\pm \text { SD }\end{array}$} & $\begin{array}{l}38.15 * \\
\pm 8.66\end{array}$ & $\begin{array}{c}54.07 * \\
\pm 7.92 \\
\end{array}$ & $\begin{array}{l}25.37 * \\
\pm 3.84\end{array}$ & $\begin{array}{c}1.66 \\
\pm 0.16 \\
\end{array}$ & $\begin{array}{c}6.74 * \\
\pm 0.75 \\
\end{array}$ & $\begin{array}{c}7.16 \\
\pm 2.39 \\
\end{array}$ & $\begin{array}{c}86.27 \\
\pm 14.22 \\
\end{array}$ & $\begin{array}{c}105.7 \\
\pm 45.53 \\
\end{array}$ \\
\hline \multicolumn{10}{|c|}{ Pinus nigra } \\
\hline \multirow{3}{*}{$\mathrm{V}$} & 44 & 211.75 & 276.13 & 136.39 & 1.68 & 1.22 & 49.80 & 77.93 & 96.8 \\
\hline & 46 & 184.11 & 247.54 & 122.19 & 1.59 & 1.30 & 42.95 & 83.79 & 110.5 \\
\hline & $\begin{array}{c}\text { Mean } \\
\pm S D\end{array}$ & $\begin{array}{l}197.93 * \\
\pm 19.54\end{array}$ & $\begin{array}{l}261.84 * \\
\pm 20.22\end{array}$ & $\begin{array}{l}129.29 * \\
\pm 10.04\end{array}$ & $\begin{array}{c}1.63 \\
\pm 0.06 \\
\end{array}$ & $\begin{aligned} & 1.26 \\
& \pm 0.06 \\
&\end{aligned}$ & $\begin{array}{l}46.38 \\
\pm 4.84 \\
\end{array}$ & $\begin{array}{r}80.86 \\
\pm 4.14 \\
\end{array}$ & $\begin{array}{r}102.6 \\
\pm 9.69 \\
\end{array}$ \\
\hline \multirow{5}{*}{ IV } & 496 & 190.58 & 233.51 & 114.96 & 1.80 & 1.59 & 37.80 & 84.96 & 80.7 \\
\hline & 504 & 192.97 & 267.13 & 131.96 & 1.61 & 1.22 & 36.30 & - & 88.5 \\
\hline & 506 & 208.87 & 294.01 & 145.25 & 1.75 & 1.21 & 34.00 & - & 88.8 \\
\hline & 507 & 234.46 & 323.62 & 160.18 & 1.63 & 1.02 & 41.60 & - & 92.1 \\
\hline & $\begin{array}{c}\text { Mean } \\
\pm S D\end{array}$ & $\begin{array}{l}203.97 * \\
\pm 20.20\end{array}$ & $\begin{array}{c}277.08 * \\
\pm 38.41\end{array}$ & $\begin{array}{l}136.86^{*} \\
\pm 19.25\end{array}$ & $\begin{array}{c}1.68 \\
\pm 0.09\end{array}$ & $\begin{array}{l}1.25 * \\
\pm 0.24\end{array}$ & $\begin{array}{l}37.43 \\
\pm 3.19\end{array}$ & $\begin{array}{l}84.96 \\
\pm 0.00\end{array}$ & $\begin{array}{c}87.5 \\
\pm 4.83\end{array}$ \\
\hline
\end{tabular}




\begin{tabular}{|c|c|c|c|c|c|c|c|c|c|}
\hline \multirow{4}{*}{ III } & 9 & 137.45 & 242.86 & 120.45 & 0.98 & 0.81 & 28.65 & 83.21 & 50.5 \\
\hline & 10 & 127.12 & 211.56 & 105.72 & 1.08 & 1.02 & 28.70 & 80.86 & 53.6 \\
\hline & 11 & 186.42 & 283.54 & 140.59 & 1.18 & 0.84 & 37.95 & 110.16 & 56.5 \\
\hline & $\begin{array}{l}\text { Mean } \\
\pm S D\end{array}$ & $\begin{array}{l}150.33 * \\
\pm 31.68\end{array}$ & $\begin{array}{l}245.98 * \\
\pm 36.09\end{array}$ & $\begin{array}{l}122.25 * \\
\pm 17.50\end{array}$ & $\begin{array}{l}1.08 * \\
\pm 0.10\end{array}$ & $\begin{array}{l}0.89 * \\
\pm 0.11\end{array}$ & $\begin{array}{l}31.77 \\
\pm 5.35\end{array}$ & $\begin{array}{c}91.41 \\
\pm 16.28\end{array}$ & $\begin{array}{c}53.5 \\
\pm 3.00\end{array}$ \\
\hline \multicolumn{2}{|c|}{$\begin{array}{c}\text { Mean } \\
\pm \text { SD }\end{array}$} & $\begin{array}{l}176.29 * \\
\pm 34.34 \\
\end{array}$ & $\begin{array}{l}257.28 * \\
\pm 34.26 \\
\end{array}$ & $\begin{array}{l}127.43^{*} \\
\pm 16.81\end{array}$ & $\begin{array}{c}1.38 \\
\pm 0.31 \\
\end{array}$ & $\begin{array}{c}1.08 * \\
\pm 0.24 \\
\end{array}$ & $\begin{array}{l}37.53 \\
\pm 6.78 \\
\end{array}$ & $\begin{array}{c}86.82 \\
\pm 11.70 \\
\end{array}$ & $\begin{array}{c}81.2 \\
\pm 21.30 \\
\end{array}$ \\
\hline \multicolumn{10}{|c|}{ Pseudotsuga menziesii } \\
\hline \multirow{3}{*}{$\mathrm{V}$} & 205 & 51.21 & 72.08 & 34.42 & 1.62 & 4.86 & 7.60 & - & 53.6 \\
\hline & 224 & 41.62 & 60.77 & 28.83 & 1.56 & 5.62 & 4.35 & 147.11 & 26.0 \\
\hline & $\begin{array}{c}\text { Mean } \\
\pm S D\end{array}$ & $\begin{array}{c}45.73^{*} \\
\pm 6.78\end{array}$ & $\begin{array}{l}65.61 * \\
\pm 8.00\end{array}$ & $\begin{array}{l}31.22 * \\
\pm 3.95\end{array}$ & $\begin{array}{c}1.58 \\
\pm 0.04\end{array}$ & $\begin{array}{c}5.24 \\
\pm 0.54\end{array}$ & $\begin{array}{c}5.98 \\
\pm 2.30\end{array}$ & $\begin{array}{l}147.11 \\
\pm 0.00\end{array}$ & $\begin{array}{c}39.8 \\
\pm 19.52\end{array}$ \\
\hline \multirow{4}{*}{ III } & 46 & 39.30 & 54.91 & 25.87 & 1.58 & 6.32 & 6.00 & 127.55 & 78.2 \\
\hline & 383 & 48.46 & 64.30 & 30.54 & 1.61 & 5.40 & 5.95 & 142.19 & 71.6 \\
\hline & 384 & 30.14 & 44.79 & 20.71 & 1.68 & 8.50 & 3.87 & 97.89 & 62.6 \\
\hline & $\begin{array}{c}\text { Mean } \\
\pm S D\end{array}$ & $\begin{array}{l}40.22 * \\
\pm 9.16\end{array}$ & $\begin{array}{l}55.63 * \\
\pm 9.76\end{array}$ & $\begin{array}{l}26.19 * \\
\pm 4.92\end{array}$ & $\begin{array}{c}1.62 \\
\pm 0.05\end{array}$ & $\begin{array}{l}6.74 * \\
\pm 1.59\end{array}$ & $\begin{array}{c}5.27 \\
\pm 1.21\end{array}$ & $\begin{array}{l}122.68 \\
\pm 22.57\end{array}$ & $\begin{array}{c}70.8 \\
\pm 7.83\end{array}$ \\
\hline \multicolumn{2}{|c|}{$\begin{array}{l}\text { Mean } \\
\pm \text { SD }\end{array}$} & $\begin{array}{l}42.48 * \\
\pm 8.28\end{array}$ & $\begin{array}{l}59.74 * \\
\pm 10.25\end{array}$ & $\begin{array}{l}28.26 * \\
\pm 5.15\end{array}$ & $\begin{array}{c}1.61 \\
\pm 0.04 \\
\end{array}$ & $\begin{array}{c}6.06 * \\
\pm 1.42\end{array}$ & $\begin{array}{c}5.55 \\
\pm 1.48\end{array}$ & $\begin{array}{c}128.79 \\
\pm 60.66\end{array}$ & $\begin{array}{c}55.3 \\
+20.35\end{array}$ \\
\hline \multicolumn{10}{|c|}{ Taxus baccata } \\
\hline \multirow{3}{*}{ V } & 58 & 39.29 & 42.80 & 19.00 & 2.40 & 12.85 & 4.45 & 98.44 & 65.8 \\
\hline & 231 & 53.64 & 47.98 & 21.21 & 2.78 & 13.28 & 5.40 & 100.08 & 49.4 \\
\hline & $\begin{array}{c}\text { Mean } \\
\pm S D\end{array}$ & $\begin{array}{l}46.47 * \\
\pm 10.15\end{array}$ & $\begin{array}{l}45.39 * \\
\pm 3.66\end{array}$ & $\begin{array}{l}20.11 * \\
\pm 1.56\end{array}$ & $\begin{array}{l}2.59 * \\
\pm 0.27\end{array}$ & $\begin{array}{l}13.06 \\
\pm 0.30\end{array}$ & $\begin{array}{c}4.93 \\
\pm 0.67\end{array}$ & $\begin{array}{l}99.26 \\
\pm 1.16\end{array}$ & $\begin{array}{c}57.6 \\
\pm 11.60\end{array}$ \\
\hline \multirow{3}{*}{ IV } & 358 & 43.21 & 42.74 & 18.84 & 2.53 & 13.52 & 5.90 & 150.21 & 100.8 \\
\hline & 791 & 34.36 & 37.63 & 16.56 & 2.26 & 14.17 & 3.45 & 143.83 & 27.1 \\
\hline & $\begin{array}{c}\text { Mean } \\
\pm S D\end{array}$ & $\begin{array}{l}38.79 * \\
\pm 6.19\end{array}$ & $\begin{array}{l}40.18^{*} \\
\pm 3.61\end{array}$ & $\begin{array}{l}17.70^{*} \\
\pm 1.61\end{array}$ & $\begin{array}{l}2.39 * \\
\pm 0.19\end{array}$ & $\begin{array}{l}13.84 \\
\pm 0.46\end{array}$ & $\begin{array}{c}4.68 \\
\pm 1.73\end{array}$ & $\begin{array}{l}147.02 \\
\pm 4.51\end{array}$ & $\begin{array}{c}63.9 \\
+52.11\end{array}$ \\
\hline \multirow{4}{*}{ III } & 379 & 41.73 & 49.65 & 22.84 & 1.99 & 8.80 & 4.30 & 130.70 & 59.6 \\
\hline & 380 & 40.85 & 41.26 & 18.35 & 2.28 & 12.80 & 3.65 & 132.71 & 64.2 \\
\hline & 381 & 38.06 & 43.32 & 19.54 & 2.12 & 10.97 & 3.80 & - & 61.8 \\
\hline & $\begin{array}{l}\text { Mean } \\
\pm S D\end{array}$ & $\begin{array}{l}40.21 \\
\pm 1.92\end{array}$ & $\begin{array}{l}44.74 * \\
\pm 4.37\end{array}$ & $\begin{array}{c}20.24^{*} \\
\pm 2.33\end{array}$ & $\begin{array}{l}2.13 * \\
\pm 0.14\end{array}$ & $\begin{array}{l}10.86^{*} \\
\pm 2.00\end{array}$ & $\begin{array}{c}3.92 \\
\pm 0.34\end{array}$ & $\begin{array}{r}131.71 \\
\pm 1.42 \\
\end{array}$ & $\begin{array}{c}61.8 \\
\pm 2.30 \\
\end{array}$ \\
\hline \multirow{4}{*}{ I } & 241 & 38.23 & 41.82 & 18.69 & 2.21 & 12.14 & 3.95 & 137.26 & 26.7 \\
\hline & 265 & 47.60 & 46.25 & 20.68 & 2.44 & 12.00 & 5.30 & 124.69 & 28.0 \\
\hline & 340 & 29.64 & 35.55 & 15.76 & 2.02 & 12.98 & 2.75 & 121.95 & 61.5 \\
\hline & $\begin{array}{c}\text { Mean } \\
\pm S D\end{array}$ & $\begin{array}{l}38.49 * \\
\pm 8.98\end{array}$ & $\begin{array}{l}41.21 * \\
\pm 5.38\end{array}$ & $\begin{array}{l}18.38 * \\
\pm 2.47\end{array}$ & $\begin{array}{l}2.22 * \\
\pm 0.21\end{array}$ & $\begin{array}{l}12.37 \\
\pm 0.53\end{array}$ & $\begin{array}{c}4.00 \\
\pm 1.27\end{array}$ & $\begin{array}{l}127.97 \\
\pm 8.16\end{array}$ & $\begin{array}{c}38.7 \\
\pm 19.73\end{array}$ \\
\hline \multicolumn{2}{|c|}{$\begin{array}{l}\text { Mean } \\
\pm \text { SD }\end{array}$} & $\begin{array}{l}40.66^{*} \\
\pm 6.66\end{array}$ & $\begin{array}{l}42.90 * \\
\pm 4.33\end{array}$ & $\begin{array}{l}19.15^{*} \\
\pm 2.09\end{array}$ & $\begin{array}{c}2.30 * \\
\pm 0.24\end{array}$ & $\begin{array}{l}12.35^{*} \\
\pm 1.53 \\
\end{array}$ & $\begin{array}{c}4.30 \\
\pm \mathbf{0 . 9 8} \\
\end{array}$ & $\begin{array}{r}126.65 \\
\pm 17.83 \\
\end{array}$ & $\begin{array}{c}54.6 \\
+22.96 \\
\end{array}$ \\
\hline
\end{tabular}

*Significant differences $(\mathrm{p}<0.05)$

\section{Morpho-physiological properties of pollen grains}

Morpho-physiological pollen properties are presented in Table 4 and Figures 5 and 6 . The impact of air pollution was calculated and graphically presented in four tree species: Cedrus atlantica, Picea omorika, Taxus baccata and Pinus nigra (Fig. 6). These four species were selected because they belong from different genera and simultaneously were present in two or more parks. The best results were obtained in the park IV (in case of P. omorika, T. baccata and P. nigra). The largest amount of pollen of $C$. atlantica was found in the park II, but its vitality (pollen tube length) was the 
highest in the park III. The parks were ranked as follows (from the best to the worst results with respect to morpho-physiological pollen properties): IV, II, III, I (only for $T$. baccata) and $\mathrm{V}$. This ranking of parks is not similar to the rankings obtained by the analysis of morphometric values of tree needles (statistic results are not presented). However, we could generally conclude that the analysed conifers have the smallest pollen grains and the lowest pollen vitality in the park with the heaviest air pollution (V) (Fig. 5). The pollens of T. baccata and C. atlantica were most sensitive to air pollution (Table 4; Fig. 5). Furthermore, P. omorika and P. nigra showed better results when situated far from the traffic zones. Small, abnormal and non-vital pollen grains lead to lower germinability and energy of pollen germination (manifested in form of small pollen tubes) (Fig. 5). The most drastic examples of changes of pollen grains were observed in the park III, in case of Cedrus atlantica and Picea omorika, and in the parks IV and V, in case of P. nigra (Table 4; Fig. 6).
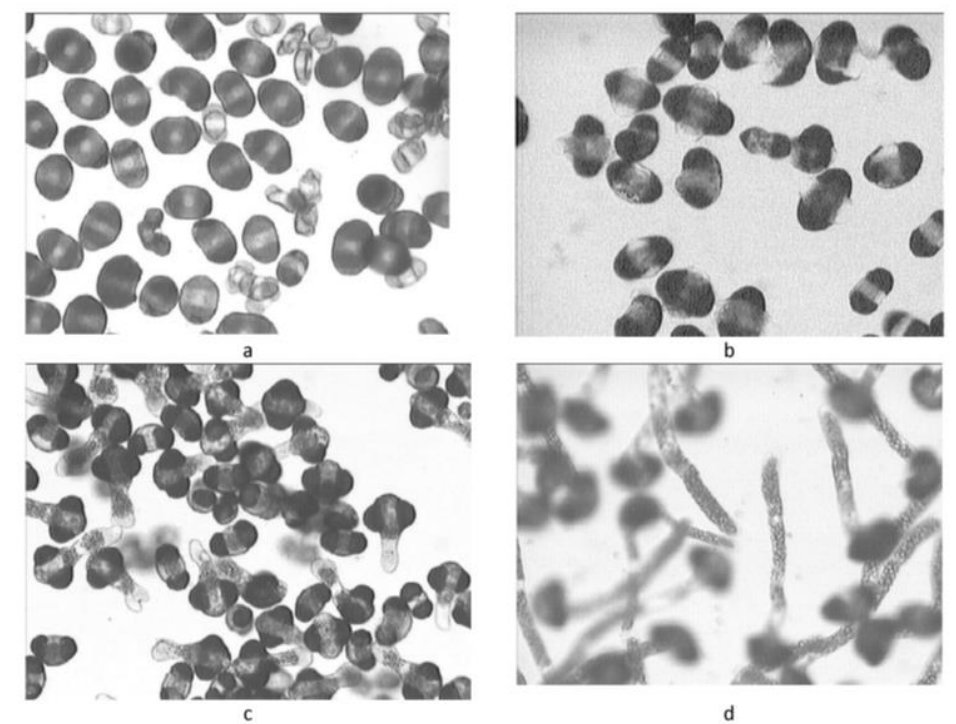

Figure 5. Small, abnormal and non-vital pollen grains of Cedrus atlantica -V (a) and Picea omorika $-V(b)$. Good germinability of Pinus nigra - IV (c) and C. atlantica - III (d) pollen grains

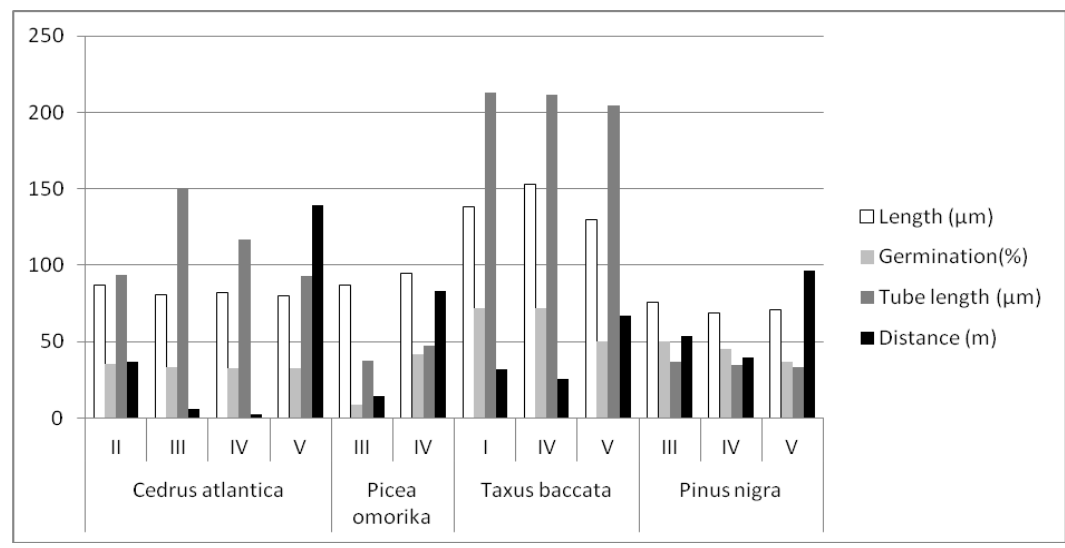

Figure 6. Graphical illustration of average values of pollen grains length, length of pollen tubes and distance from traffic in Cedrus atlantica, Picea omorika, Taxus baccata, and Pinus nigra 
In some analyzed species distances of particular trees from heavy traffic also were in significant correlation with needle dimensions (A. concolor, A. nordmanniana and $P$. omorika, $\mathrm{r}=-0.93,0.99$, and 0.56, respectively, Fig. 7), stomatal density (A. alba, P. abies, $P$. omorika, $P$. pungens, $\mathrm{r}=-0.99,1.0,-0.70$, and 0.71 , resp., Fig. 8 ) and pollen vitality (germinability: $C$. atlantica, $\mathrm{r}=0.73, P$. nigra, $\mathrm{r}=0.57$ and $T$. baccata, $\mathrm{r}=0.68$, Fig. 9; length of pollen tubes: A. pinsapo, $\mathrm{r}=0.99$ and $P$. menziesii, $\mathrm{r}=0.95$, Fig. 10).

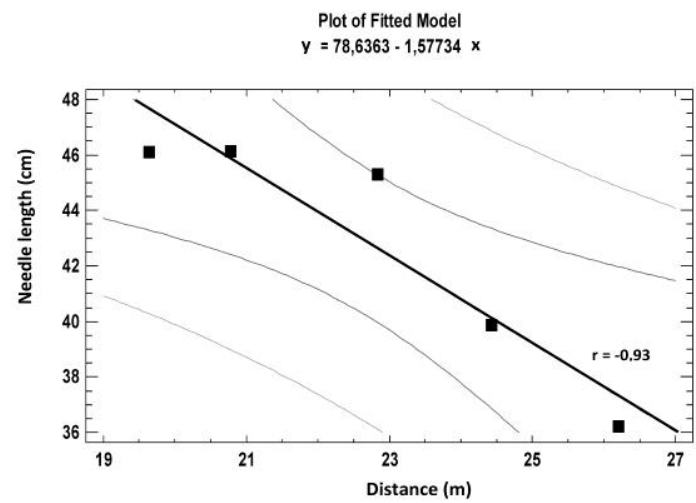

$\mathbf{a}$

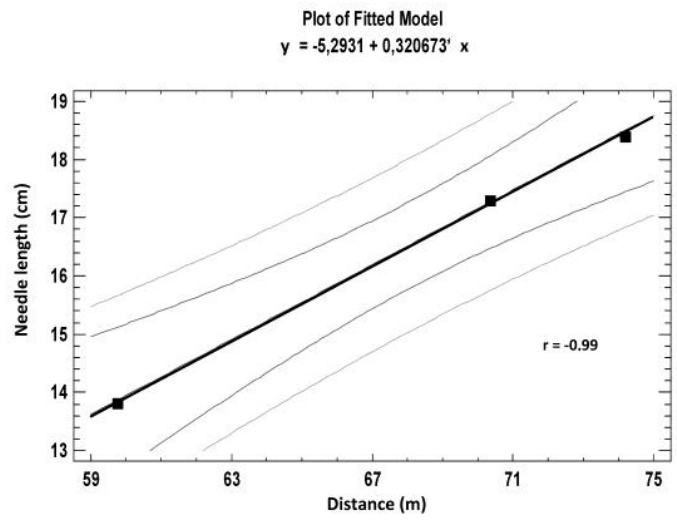

b

Plot of Fitted Model $y=19,1801+0,0282816 \times$

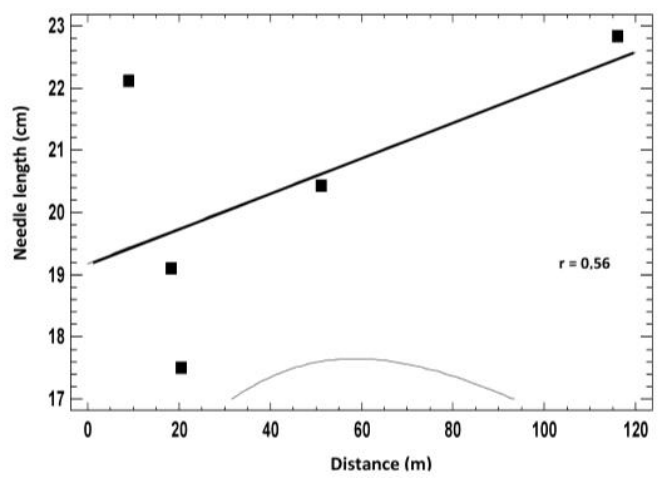

c

Figure 7. Correlation between needle length and distance from traffic in Abies concolor (a), A. nordmanniana (b) and Picea omorika (c)

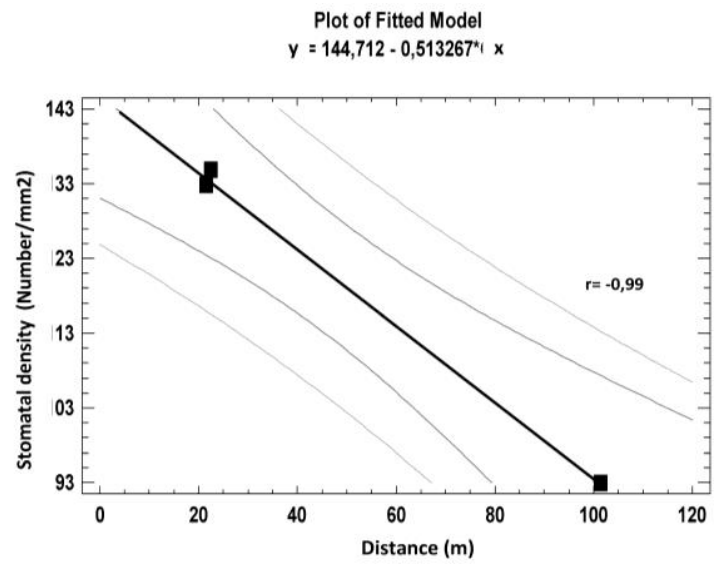

a
Plot of Fitted Model

$y=15,7044+1,59444^{*} \times$

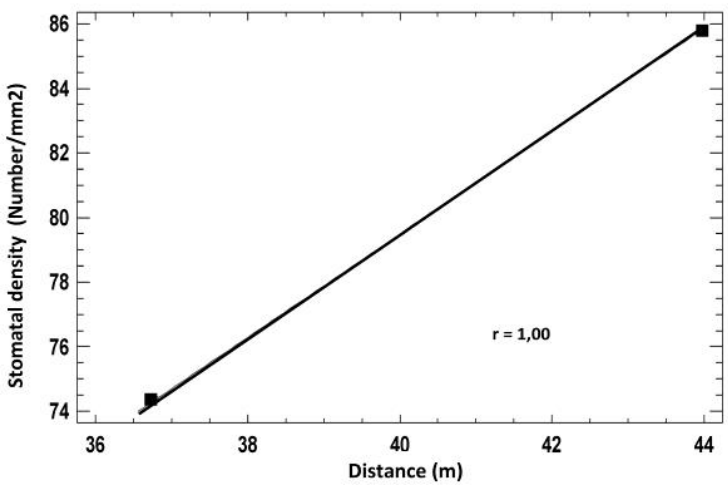

b 


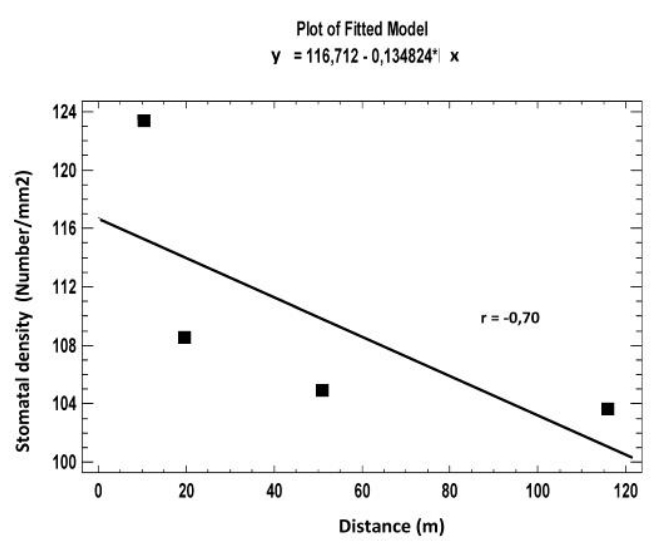

c

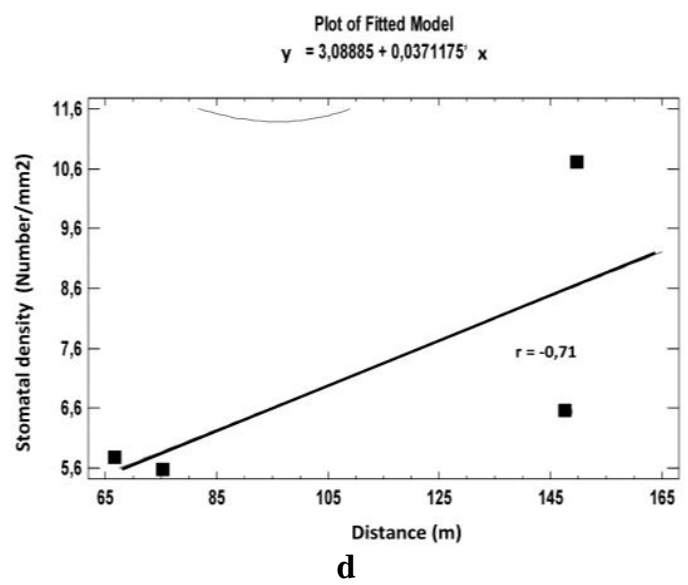

d

Figure 8. Correlation between stomatal density and distance from traffic in Abies alba (a), Picea abies (b), Picea omorika (c) and P. pungens (d)

Table 4. Results of morpho-physiological analysis of pollen grains and distance of trees from traffic

\begin{tabular}{|c|c|c|c|c|c|c|c|}
\hline \multirow{4}{*}{ Park No. } & \multirow{4}{*}{ Tree No. } & \multicolumn{5}{|c|}{ Pollen analyses } & \multirow{4}{*}{$\begin{array}{l}\text { Distance of } \\
\text { trees from } \\
\text { traffic }(\mathbf{m})\end{array}$} \\
\hline & & \multicolumn{3}{|c|}{ Morphological } & \multirow{2}{*}{\multicolumn{2}{|c|}{$\begin{array}{c}\text { Physiological } \\
\text { Vitality }\end{array}$}} & \\
\hline & & \multicolumn{2}{|c|}{ Dimensions } & \multirow[b]{2}{*}{$\begin{array}{c}\text { Shape } \\
(\%)\end{array}$} & & & \\
\hline & & $\begin{array}{c}\text { Length } \\
(\mu \mathrm{m})\end{array}$ & $\begin{array}{l}\text { Width } \\
(\mu \mathrm{m})\end{array}$ & & $\begin{array}{c}\text { Germination } \\
(\%)\end{array}$ & $\begin{array}{c}\text { Length of } \\
\text { pollen } \\
\text { tubes }(\mu \mathrm{m}) \\
\end{array}$ & \\
\hline \multicolumn{8}{|c|}{ Abies alba } \\
\hline III & 96 & 149.47 & 103.31 & 69.45 & 48.45 & 39.77 & 100.3 \\
\hline \multicolumn{2}{|c|}{$\begin{array}{l}\text { Mean } \\
\pm \text { SD }\end{array}$} & $\begin{array}{l}149.47 \\
\pm 0.00 \\
\end{array}$ & $\begin{array}{c}103.31 \\
\pm 0.00 \\
\end{array}$ & $\begin{array}{r}69.45 \\
\pm 0.00 \\
\end{array}$ & $\begin{array}{r}48.45 \\
\pm 0.00 \\
\end{array}$ & $\begin{array}{r}39.77 \\
\pm \mathbf{0 . 0 0} \\
\end{array}$ & $\begin{array}{r}100.3 \\
\pm 0.00 \\
\end{array}$ \\
\hline \multicolumn{8}{|c|}{ Abies nordmanniana } \\
\hline \multirow{2}{*}{ I } & 149 & 123.76 & 88.66 & 72.17 & 62.91 & 44.59 & 74.1 \\
\hline & 150 & 139.73 & 96.07 & 69.01 & 51.27 & 52.01 & 70.3 \\
\hline \multicolumn{2}{|c|}{$\begin{array}{l}\text { Mean } \\
\pm \text { SD }\end{array}$} & $\begin{array}{l}131.75 * \\
\pm 11.29\end{array}$ & $\begin{array}{l}92.36 * \\
\pm 5.24\end{array}$ & $\begin{array}{l}70.59 * \\
\pm 2.23\end{array}$ & $\begin{array}{r}\mathbf{5 7 . 0 9} \\
\pm \mathbf{8 . 2 3} \\
\end{array}$ & $\begin{array}{l}47.74 * \\
\pm 5.25\end{array}$ & $\begin{array}{c}72.2 \\
\pm 2.69 \\
\end{array}$ \\
\hline \multicolumn{8}{|c|}{ Abies pinsapo } \\
\hline \multirow{3}{*}{ IV } & 8 & 130.16 & 95.40 & 73.63 & 47.00 & 27.15 & 25.6 \\
\hline & 373 & 142.39 & 95.86 & 68.17 & 46.81 & 51.03 & 96.8 \\
\hline & 375 & 138.80 & 94.38 & 67.39 & 60.26 & 50.57 & 92.2 \\
\hline \multicolumn{2}{|c|}{$\begin{array}{l}\text { Mean } \\
\pm \text { SD }\end{array}$} & $\begin{array}{c}137.12 * \\
\pm 6.29\end{array}$ & $\begin{array}{c}95.21 \\
\pm 0.76 \\
\end{array}$ & $\begin{array}{c}69.73 * \\
\pm 3.40 \\
\end{array}$ & $\begin{array}{r}51.36 \\
\pm 7.71 \\
\end{array}$ & $\begin{array}{l}42.92 * \\
\pm 13.66 \\
\end{array}$ & $\begin{array}{c}74.8 \\
\pm 39.85 \\
\end{array}$ \\
\hline \multicolumn{8}{|c|}{ Cedrus atlantica } \\
\hline \multirow{3}{*}{$\mathrm{V}$} & 26 & 79.00 & 54.13 & 68.60 & 18.36 & 71.38 & 86.8 \\
\hline & 80 & 81.27 & 60.50 & 74.70 & 24.13 & 108.85 & 44.3 \\
\hline & $\begin{array}{c}\text { Mean } \\
\pm S D\end{array}$ & $\begin{array}{c}80.14 \\
\pm 1.60 \\
\end{array}$ & $\begin{array}{l}57.32 * \\
\pm 4.50\end{array}$ & $\begin{array}{c}71.65^{*} \pm \\
4.31\end{array}$ & $\begin{array}{l}21.25 * \\
\pm 4.08\end{array}$ & $\begin{array}{l}92.79 * \\
\pm 26.50\end{array}$ & $\begin{array}{l}67.0 \\
+30.05\end{array}$ \\
\hline \multirow{4}{*}{ IV } & 59 & 83.03 & 52.73 & 63.66 & 31.40 & 110.39 & 148.2 \\
\hline & 68 & 80.64 & 54.99 & 68.43 & 32.99 & 111.46 & 161.9 \\
\hline & 83 & 82.27 & 50.86 & 61.90 & 52.53 & 128.37 & 133.1 \\
\hline & $\begin{array}{c}\text { Mean } \\
\pm S D\end{array}$ & $\begin{array}{l}81.98 \\
\pm 1.22\end{array}$ & $\begin{array}{l}52.86^{*} \\
\pm 2.07\end{array}$ & $\begin{array}{l}64.66^{*} \\
\pm 3.38\end{array}$ & $\begin{array}{l}38.97 * \\
\pm 12.03\end{array}$ & $\begin{array}{l}116.94 * \\
\pm 10.09\end{array}$ & $\begin{array}{c}147.7 \\
\pm 14.41\end{array}$ \\
\hline
\end{tabular}




\begin{tabular}{|c|c|c|c|c|c|c|c|}
\hline \multirow{4}{*}{ III } & 322 & 79.02 & 51.11 & 64.78 & 3.70 & 101.90 & 10.8 \\
\hline & 329 & 84.26 & 56.70 & 67.46 & 33.58 & 157.80 & 32.2 \\
\hline & 335 & 78.04 & 53.17 & 74.20 & 12.86 & 174.34 & 34.9 \\
\hline & $\begin{array}{c}\text { Mean } \\
\pm S D\end{array}$ & $\begin{array}{l}80.44 * \\
\pm 3.34 \\
\end{array}$ & $\begin{array}{l}53.66^{*} \\
\pm 2.83 \\
\end{array}$ & $\begin{array}{l}68.81 * \\
\pm 4.85 \\
\end{array}$ & $\begin{array}{l}16.72 * \\
\pm 15.31 \\
\end{array}$ & $\begin{array}{l}150.33 * \\
\pm 37.96 \\
\end{array}$ & $\begin{array}{r}26.9 \\
\pm 13.20 \\
\end{array}$ \\
\hline \multirow[b]{2}{*}{ II } & 47 & 86.91 & 55.69 & 64.17 & 3.51 & 93.79 & 4.5 \\
\hline & $\begin{array}{c}\text { Mean } \\
\pm S D\end{array}$ & $\begin{array}{l}86.91 \\
\pm 0.00\end{array}$ & $\begin{array}{l}55.69 \\
\pm 0.00\end{array}$ & $\begin{array}{c}64.17 \\
\pm 0.00\end{array}$ & $\begin{array}{c}3.51 \\
\pm 0.00\end{array}$ & $\begin{array}{c}93.79 \\
\pm 0.00\end{array}$ & $\begin{array}{c}4.5 \\
\pm 0.00\end{array}$ \\
\hline \multicolumn{2}{|c|}{$\begin{array}{l}\text { Mean } \\
\pm \text { SD } \\
\end{array}$} & $\begin{array}{l}81.61 * \\
\pm 2.85 \\
\end{array}$ & $\begin{array}{l}54.43 * \\
\pm 3.00 \\
\end{array}$ & $\begin{array}{l}67.54 \% \\
\pm 4.52 \\
\end{array}$ & $\begin{array}{l}23.67 * \\
\pm 15.85 \\
\end{array}$ & $\begin{array}{l}119.82 * \\
\pm 31.75 \\
\end{array}$ & $\begin{array}{r}61.5 \\
\pm 61.09 \\
\end{array}$ \\
\hline \multicolumn{8}{|c|}{ Picea abies } \\
\hline III & 382 & 114.54 & 74.07 & 64.63 & 24.59 & 75.36 & 62.1 \\
\hline \multicolumn{2}{|c|}{$\begin{array}{c}\text { Mean } \\
\pm \text { SD } \\
\end{array}$} & $\begin{array}{l}114.54 \\
\pm 0.00 \\
\end{array}$ & $\begin{array}{r}74.07 \\
\pm 0.00 \\
\end{array}$ & $\begin{array}{r}64.63 \\
\pm 0.00 \\
\end{array}$ & $\begin{array}{r}24.59 \\
\pm 0.00 \\
\end{array}$ & $\begin{array}{r}75.36 \\
\pm 0.00 \\
\end{array}$ & $\begin{array}{c}62.1 \\
\pm 0.00 \\
\end{array}$ \\
\hline \multicolumn{8}{|c|}{ Picea omorika } \\
\hline \multirow{4}{*}{ IV } & 362 & 94.91 & 57.85 & 61.18 & 28.19 & 36.89 & 115.9 \\
\hline & 367 & 96.99 & 56.43 & 58.31 & 19.54 & 53.26 & 82.4 \\
\hline & 495 & 92.50 & 52.84 & 57.58 & 77.30 & 46.59 & 51.2 \\
\hline & $\begin{array}{c}\text { Mean } \\
\pm S D \\
\end{array}$ & $\begin{array}{l}94.80 * \\
\pm 2.25\end{array}$ & $\begin{array}{l}55.71 * \\
\pm 2.58\end{array}$ & $\begin{array}{l}59.02 * \\
\pm 1.90\end{array}$ & $\begin{array}{l}41.68 * \\
\pm 31.15\end{array}$ & $\begin{array}{l}47.61 * \\
\pm 8.23\end{array}$ & $\begin{array}{c}83.1 \\
+32.36 \\
\end{array}$ \\
\hline \multirow{3}{*}{ III } & 1 & 86.97 & 53.70 & 61.94 & 2.88 & 27.0 & 9.3 \\
\hline & 4 & 86.94 & 56.23 & 64.94 & 14.51 & 37.82 & 19.7 \\
\hline & $\begin{array}{c}\text { Mean } \\
\pm S D\end{array}$ & $\begin{array}{l}86.95 \\
\pm 0.02 \\
\end{array}$ & $\begin{array}{l}54.96 * \\
\pm 1.79 \\
\end{array}$ & $\begin{array}{l}63.44 * \\
\pm 2.12 \\
\end{array}$ & $\begin{array}{l}8.70 * \\
\pm 8.22 \\
\end{array}$ & $\begin{array}{l}32.41 * \\
\pm 7.65 \\
\end{array}$ & $\begin{aligned} & 14.5 \\
& \pm 7.35 \\
&\end{aligned}$ \\
\hline \multicolumn{2}{|c|}{$\begin{array}{l}\text { Mean } \\
\pm \text { SD } \\
\end{array}$} & $\begin{array}{l}91.66 * \\
\pm 4.58 \\
\end{array}$ & $\begin{array}{l}55.41 \\
\pm 2.07 \\
\end{array}$ & $\begin{array}{c}60.79 * \\
\pm 2.96 \\
\end{array}$ & $\begin{array}{c}28.48 * \pm \\
28.78 \\
\end{array}$ & $\begin{array}{c}40.31 * \pm \\
10.03 \\
\end{array}$ & $\begin{array}{c}55.7 \\
\pm 44.17 \\
\end{array}$ \\
\hline \multicolumn{8}{|c|}{ Pinus nigra } \\
\hline \multirow{4}{*}{$\mathrm{V}$} & 39 & 73.21 & 45.04 & 61.61 & 83.51 & 37.28 & 101.0 \\
\hline & 45 & 72.19 & 43.96 & 60.96 & 78.27 & 32.81 & 95.1 \\
\hline & 46 & 68.23 & 43.34 & 63.60 & 36.67 & 27.65 & 93.1 \\
\hline & $\begin{array}{c}\text { Mean } \\
\pm S D\end{array}$ & $\begin{array}{l}71.21 * \\
\pm 2.63 \\
\end{array}$ & $\begin{array}{l}44.11 \\
\pm 0.86 \\
\end{array}$ & $\begin{array}{l}62.06 * \\
\pm 1.37 \\
\end{array}$ & $\begin{array}{l}66.15 * \\
\pm 25.66 \\
\end{array}$ & $\begin{array}{l}33.48 * \\
\pm 4.82 \\
\end{array}$ & $\begin{array}{c}96.4 \\
\pm 4.11 \\
\end{array}$ \\
\hline \multirow{4}{*}{ IV } & 2 & 69.28 & 46.76 & 67.64 & 63.02 & 41.51 & 41.6 \\
\hline & 4 & 64.60 & 40.53 & 62.98 & 22.71 & 27.45 & 34.9 \\
\hline & 6 & 72.48 & 44.92 & 62.22 & 50.56 & 33.37 & 44.0 \\
\hline & $\begin{array}{c}\text { Mean } \\
\pm S D\end{array}$ & $\begin{array}{l}68.78 * \\
\pm 3.96 \\
\end{array}$ & $\begin{array}{l}4.07 * \\
\pm 3.20 \\
\end{array}$ & $\begin{array}{l}64.28 * \\
\pm 2.93 \\
\end{array}$ & $\begin{array}{l}45.43 * \\
\pm 20.64 \\
\end{array}$ & $\begin{array}{l}35.11 * \\
\pm 7.06 \\
\end{array}$ & $\begin{array}{c}40.1 \\
\pm 4.72 \\
\end{array}$ \\
\hline \multirow{4}{*}{ III } & 9 & 79.42 & 52.60 & 66.42 & 52.49 & 38.46 & 50.5 \\
\hline & 10 & 69.15 & 43.83 & 63.35 & 42.74 & 28.81 & 53.6 \\
\hline & 11 & 78.70 & 51.00 & 64.89 & 54.41 & 40.65 & 56.5 \\
\hline & $\begin{array}{c}\text { Mean } \\
\pm S D \\
\end{array}$ & $\begin{array}{l}75.76 * \\
\pm 5.73 \\
\end{array}$ & $\begin{array}{l}49.14 * \\
\pm 4.67 \\
\end{array}$ & $\begin{array}{l}64.89 * \\
\pm 1.53 \\
\end{array}$ & $\begin{array}{r}49.88 \\
\pm 6.26 \\
\end{array}$ & $\begin{array}{l}37.18 * \\
\pm 6.30 \\
\end{array}$ & $\begin{array}{c}52.2 \\
\pm 3.00 \\
\end{array}$ \\
\hline \multicolumn{2}{|c|}{$\begin{array}{l}\text { Mean } \\
\pm \text { SD }\end{array}$} & $\begin{array}{l}71.92 * \\
\pm 4.82\end{array}$ & $\begin{array}{l}\text { 45.78* } \\
\pm 3.82 \\
\end{array}$ & $\begin{array}{c}63.74 * \\
\pm 2.21 \\
\end{array}$ & $\begin{array}{l}53.82 * \\
\pm 19.24 \\
\end{array}$ & $\begin{array}{l}34.92 * \\
\pm 5.51\end{array}$ & $\begin{array}{c}46.1 \\
+25.68 \\
\end{array}$ \\
\hline \multicolumn{8}{|c|}{ Pseudotsuga menziessi } \\
\hline \multirow[b]{2}{*}{$\mathrm{V}$} & 205 & 97.98 & 85.37 & 87.71 & 76.58 & 140.19 & 53.6 \\
\hline & $\begin{array}{c}\text { Mean } \\
\pm S D \\
\end{array}$ & $\begin{array}{l}97.98 \\
\pm 0.00 \\
\end{array}$ & $\begin{array}{l}85.37 \\
\pm 0.00 \\
\end{array}$ & $\begin{array}{l}87.71 \\
\pm 0.00 \\
\end{array}$ & $\begin{array}{l}76.58 \\
\pm 0.00 \\
\end{array}$ & $\begin{array}{r}140.19 \\
\pm 0.00 \\
\end{array}$ & $\begin{array}{l}53.6 \\
\pm 0.00 \\
\end{array}$ \\
\hline \multirow{2}{*}{ III } & 46 & 126.56 & 100.32 & 80.02 & 67.42 & 147.70 & 78.2 \\
\hline & 383 & 132.76 & 102.78 & 78.48 & 54.97 & 143.64 & 71.6 \\
\hline
\end{tabular}




\begin{tabular}{|c|c|c|c|c|c|c|c|}
\hline & 384 & 144.74 & 102.60 & 71.80 & 80.16 & 142.62 & 62.6 \\
\hline & $\begin{array}{l}\text { Mean } \\
\pm S D\end{array}$ & $\begin{array}{c}135.94 * \\
\pm 9.24\end{array}$ & $\begin{array}{l}102.14 \\
\pm 1.37\end{array}$ & $\begin{array}{l}76.27 * \\
\pm 4.37\end{array}$ & $\begin{array}{l}67.51 * \\
\pm 12.59\end{array}$ & $\begin{array}{l}144.65 \\
\pm 2.69\end{array}$ & $\begin{array}{c}70.8 \\
\pm 7.83\end{array}$ \\
\hline \multicolumn{2}{|c|}{$\begin{array}{l}\text { Mean } \\
\pm \text { SD }\end{array}$} & $\begin{array}{l}125.40 * \\
\pm 19.84\end{array}$ & $\begin{array}{l}97.49 * \\
\pm 8.34\end{array}$ & $\begin{array}{l}79.44 * \\
\pm 6.53\end{array}$ & $\begin{array}{c}69.78 * \\
\pm 11.24\end{array}$ & $\begin{array}{l}143.54 \\
\pm 3.13\end{array}$ & $\begin{array}{c}62.2 \\
\pm 10.72\end{array}$ \\
\hline \multicolumn{8}{|c|}{ Taxus baccata } \\
\hline \multirow{3}{*}{$\mathrm{V}$} & 242 & 111.26 & 99.32 & 89.56 & 50.50 & 194.81 & 66.2 \\
\hline & 243 & 148.42 & 129.00 & 86.30 & 49.88 & 214.13 & 67.4 \\
\hline & $\begin{array}{c}\text { Mean } \\
\pm S D\end{array}$ & $\begin{array}{l}129.84 * \\
\pm 26.28\end{array}$ & $\begin{array}{l}113.71 * \\
\pm 20.99\end{array}$ & $\begin{array}{l}87.93 * \\
\pm 2.30\end{array}$ & $\begin{array}{l}50.19 \\
\pm 0.44\end{array}$ & $\begin{array}{l}204.47 \\
\pm 13.66\end{array}$ & $\begin{array}{l}66.8 m \\
\pm 0.85\end{array}$ \\
\hline \multirow{4}{*}{ IV } & 791 & 150.07 & 131.50 & 87.70 & 75.40 & 201.62 & 27.1 \\
\hline & 792 & 179.32 & 156.71 & 87.68 & 69.82 & 215.82 & 25.0 \\
\hline & 793 & 129.89 & 116.56 & 90.25 & 71.02 & 216.94 & 24.7 \\
\hline & $\begin{array}{c}\text { Mean } \\
\pm S D\end{array}$ & $\begin{array}{l}153.10^{*} \\
\pm 24.85\end{array}$ & $\begin{array}{l}134.89 * \\
\pm 20.29\end{array}$ & $\begin{array}{l}88.54 \\
\pm 1.48\end{array}$ & $\begin{array}{l}72.08 \\
\pm 2.94\end{array}$ & $\begin{array}{l}211.46 \\
\pm 8.54\end{array}$ & $\begin{array}{c}25.6 \\
\pm 1.31\end{array}$ \\
\hline \multirow{3}{*}{ I } & 340 & 134.72 & 115.35 & 86.60 & 74.68 & 213.03 & 61.5 \\
\hline & 669 & 139.98 & 124.83 & 89.73 & 69.76 & 212.78 & 3.2 \\
\hline & $\begin{array}{c}\text { Mean } \\
\pm S D\end{array}$ & $\begin{array}{l}138.23 \\
\pm 3.72\end{array}$ & $\begin{array}{l}121.67 \\
\pm 6.70\end{array}$ & $\begin{array}{l}88.69 \\
\pm 2.21\end{array}$ & $\begin{array}{l}72.22 \\
\pm 3.48\end{array}$ & $\begin{array}{l}212.91 \\
\pm 0.18\end{array}$ & $\begin{array}{c}32.3 \\
\pm 41.22\end{array}$ \\
\hline \multicolumn{2}{|c|}{$\begin{array}{l}\text { Mean } \\
\pm \text { SD }\end{array}$} & $\begin{array}{l}142.72 * \\
\pm 21.01\end{array}$ & $\begin{array}{l}125.50 * \\
\pm 17.73\end{array}$ & $\begin{array}{r}\mathbf{8 8 . 3 7} \\
\pm \mathbf{1 . 5 8}\end{array}$ & $\begin{array}{c}65.87 * \\
\pm 10.94\end{array}$ & $\begin{array}{c}209.88 \\
\pm 8.33\end{array}$ & $\begin{array}{r}38.2 \\
\pm 25.41\end{array}$ \\
\hline
\end{tabular}

*Significant differences $(p<0.05)$

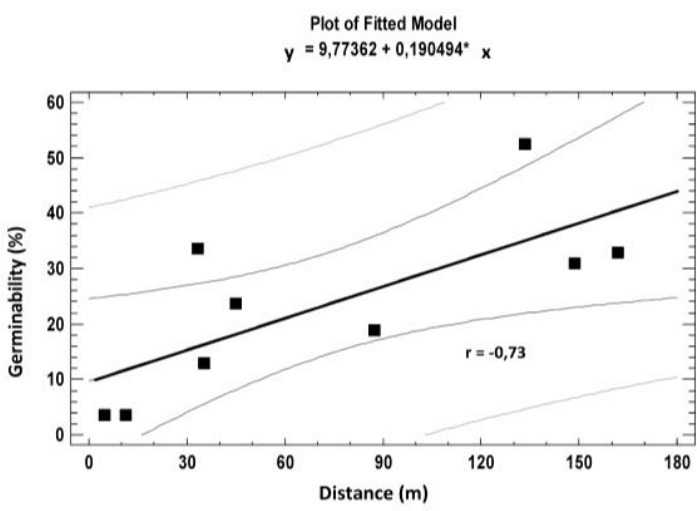

a

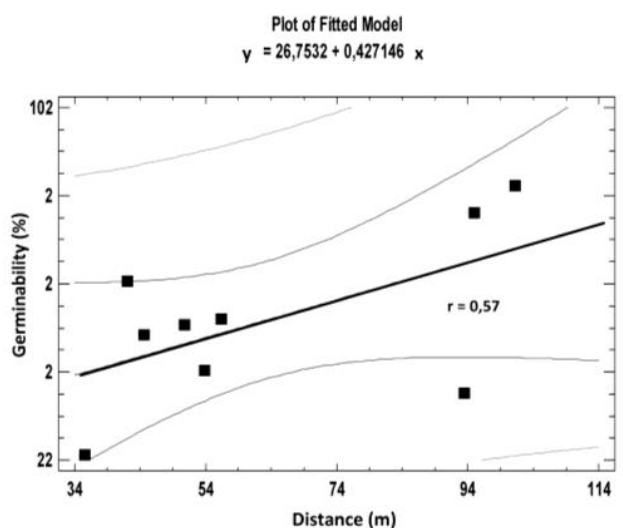

b

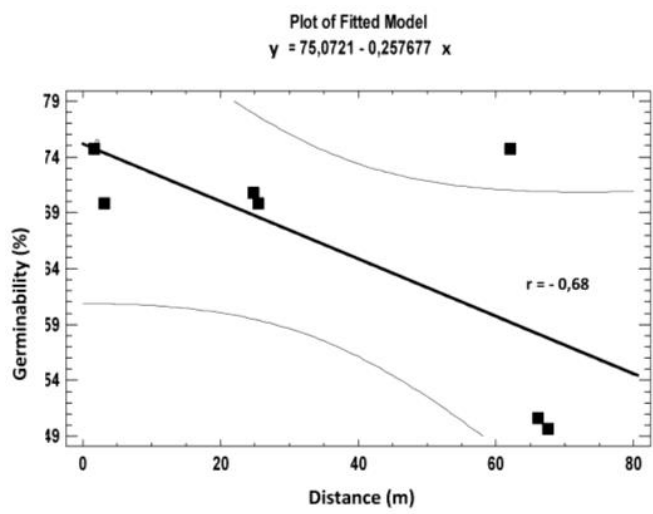

c

Figure 9. Correlation between germinability and distance from traffic in Cedrus atlantica (a), Pinus nigra (b), and Taxus baccata (c) 


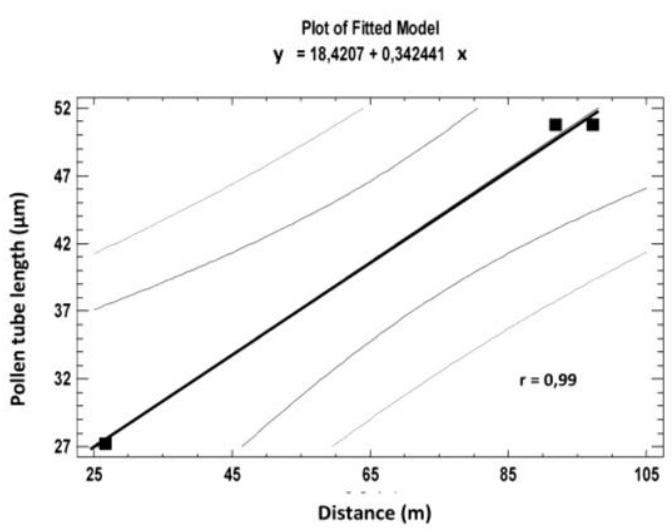

a

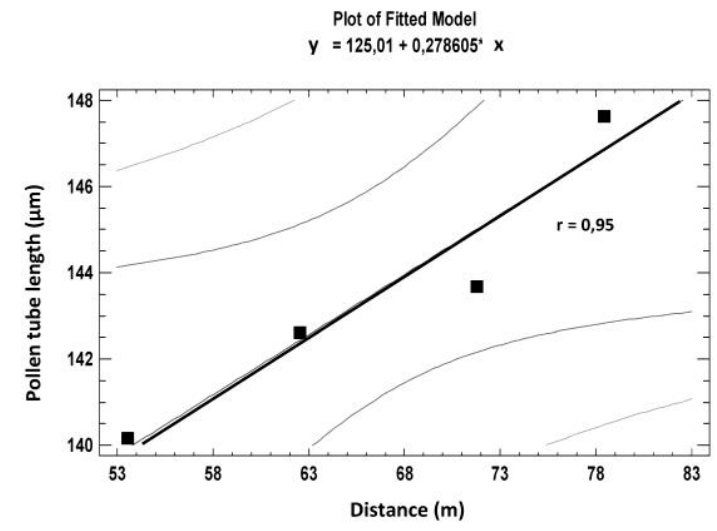

b

Figure 10. Correlation between pollen tube length and distance from traffic in Abies pinsapo (a), and Pseudotsuga menziesii (b)

\section{Discussion}

Conifer foliage is a useful indicator in bio-monitoring of air pollution (Pinus ponderosa). Several of investigated species Vukićević (1987) considered resistant to urban conditions (A. concolor, A. nordmanianna, A. pinsapo, $C$. atlantica and $P$. omorika). The average needle length and width for all twelve investigated species are in agreement with the published results of Vidaković (1982) and Vukićević (1987). The needle length and width of $A$. alba from park $\mathrm{V}$ also agree with previous results obtained by Pawlaczyk et al. (2005) and Pawlaczyk and Bobowicz (2008). The average needle length and width of $P$. omorika are also in agreement with the results obtained in natural habitats (Milovanović et al., 2005; Radovanović et al., 2014; Nikolić et al., 2015). Furthermore, dimensions of Pinus nigra from park V are in agreement with the results obtained by Matziris (1984) and Borzan et al. (2002). The values of needle length and leaf area in our A. pinsapo from park IV are higher than those already reported by Sękiewicz et al. (2013). The needle length of Cedrus atlantica also agrees with the results obtained by Jasińska et al. (2013), while needle width was about $25 \%$ higher. Needle width of Pinus sylvestris was higher in $\mathrm{CO}_{2}$ polluted air, as a result of an increase of messophyl tissue (Lin et al., 2001). In some other conifers, air-polluted needles are shorter, with lower number of stomata (Pinus pinaster, Wahid et al., 2006). Effects of drought could be found even at the level of needle anatomy, where stomatal density increased as needle length decreased (Pinus canariensis, Grill et al., 2004).

Dimensions of trees and their leaves could depend on soil nutrition. Addition of nitrogen increased length, width and number of needles per shoot in P. menziesii (Brix and Ebell, 1969).

In A. alba, A. nordmanniana and A. pinsapo stomatal density was higher (Table 1) than in literature (Robakowski et al., 2004; Meidner and Mansfield, 1968; SanchoKnapik et al., 2014, resp). The same situation was found in P. abies and P. pungens (Dixon et al., 1995 and Meidner and Mansfield, 1968, resp.). In T. baccata stomatal density is lower in park $\mathrm{V}$ than in other investigated parks, but similar with literature results (Stefanović, 2015).

Our results of pollen length of $P$. abies and $P$. omorika were slightly higher than those reported by Jia et al. (2014), but germination was significantly low, especially of $P$. omorika in park III (Table 2). In our previous investigations germination of $P$. 
omorika pollen was 54-68\% (max. 94\%) (Batos and Nikolić, 2013). In case of $P$. menziesii, the average values of pollen size were similar to those reported in the literature (Ho, 1968). The air pollution changed the properties of leaves and pollen grains through chemical changes. An increased content of lead in the air influenced lower pollen germination and pollen tube growth in Pinus strobus and P. resinosa (Cox, 1988). In case of Abies alba, contaminated pollen decreased vitality and germination up to $50 \%$, which depended on genotype-specific response to air pollution (Kormuták, 1996 and refs. cited therein). The same situation is found in our results of A. alba (Table 2), where decreased germinability and length of pollen tubes was also up to $60 \%$ and $50 \mu \mathrm{m}$, respectively. Furthermore, in some species ( $P$. omorika, P. nigra, etc.) we found some abnormalities in dimensions, shape and vitality of pollen grains, which occurred slightly more frequently in more heavily contaminated parks (case of $C$. atlantica, P. omorika, etc., Table 2, Figs. 5 and 6 ).

We have to underline that presented results of morpho-anatomical and pollen propertiesof conifers in urban area have never been explored so detailed in the past.

\section{Conclusions}

Based on the presented results of morpho-anatomical and morpho-physiological characteristics of conifer needles and pollen, respectively, it can be concluded that the consequences of air pollution are more apparent in case of pollen. In city parks with heavy traffic (V, IV, III), pollen grains of trees were smaller and less vital. Very often results were in correlation with distance of trees from traffic. Some of examined conifer species are especially sensitive to air pollution caused by vehicles (Picea omorika, Cedrus atlantica, Taxus baccata, Pinus nigra), especially in pollen properties. The level of air contamination, as well as other atmospheric conditions (temperature, humidity, wind direction and strength, etc.) could vary among different years, too. All the above factors and their influence on growth, development and blooming of trees, should be taken into consideration in landscape architecture and horticulture works in future.

Acknowledgements. This work was supported by Ministry of Education, Science and Technological Development of Serbia (grant numbers 173029, 173011, 31070 and 43007), as well as by City of Belgrade, Secretariat for Environmental Protection (grant number 4011-144).

\section{REFERENCES}

[1] Apple, M. E., Olszyk, D. M., Ormrod, D. P., Lewis, J., Southworth, D., Tingey, D. T. (2000): Morphology and stomatal function of Douglas-fir needles exposed to climate change: elevated CO2 and temperature. - International Journal of Plant Sciences 161: 127-132.

[2] Batos, B. Ž., Nikolić, B. M. (2013): Variability of in vitro germination of Picea omorika pollen. - Dendrobiology 69: 13-19.

[3] Borzan, Ž., Idžojtić, M., Guttenberger, H. (2002): Identification of F1 hybrids Pinus nigra J. F. Arnold $x$ P. sylvestris L., P. nigra J. F. Arnold $x$ P. densiflora Siebold et Zucc. and $P$. nigra J. F. Arnold $x \quad P$. thunbergiana Franco by internal and external morphometric traits. - Silvae Genetica 51: 270-273.

[4] Brix, H., Ebell, L. F. (1969): Effects of nitrogen fertilization on growth, leaf area, and photosynthesis rate in Douglas-Fir. - Forest Science 15: 189-196. 
[5] Cox, R. M. (1988): The sensitivity of pollen from various coniferous and broad-leaved trees to combinations of acidity and trace metals. - New Phytologist 109: 193-201.

[6] Dixon, M., Le Thiec, D., Garrec, J.-P. (1995): The growth and gass-exchange response of soil-planted Norway spruce (Picea abies (L.) Karst.) and red oak (Quercus rubra L.) exposed to elevated $\mathrm{CO}_{2}$ and to naturally occuring drought. - New Phytologist 129: 265273.

[7] Donovan, R. G., Stewart, H. E., Owen, S. M., MacKenzie, A. R., Hewitt, C. N. (2005): Development and application of an urban tree air quality score for photochemical pollution episodes using the Birmingham, United Kingdom, area as a case study. Environmental Science \& Technology 39: 6730-6738.

[8] Freer-Smith, P. H., Beckett, K. P., Taylor, G. (2005): Deposition velocities to Sorbus aria, Acer campestre, 'Beaupre', Pinus nigra and $x$ Cupressocyparis leylandii for coarse, fine and ultra-fine particles in the urban environment. - Environmental Pollution 133: 157-167.

[9] Grill, D., Tausz, M., Pöllinger, U., Jimenez, M. S., Morales, D. (2004): Effects of drought on needle anatomy of Pinus canariensis. - Flora 199: 85-89.

[10] Hällgren, J.-E., Fredriksson, S.-Å. (1982): Emission of hydrogen sulfide from sulfur dioxide-fumigated Pine trees. - Plant Physiology 70: 456-459.

[11] Ho, R. (1968): Some observations on germination of Pseudotsuga menziessi (Mirb.) Franco pollen in vitro. - Master of Science Thesis, The University of British Columbia.

[12] Jasińska, A. K., Boratyńska, K., Sobierajska, K., Romo, A., Ok, T., Kharat, M. B. D., Boratyński, A. (2013): Relationships among Cedrus libani, C. brevifolia and C. atlantica as revealed by the morphological and anatomical needle characters. - Plant Systematics and Evolution 200: 35-48.

[13] Jia, Z. R., Wang, J. H., Zhang, S. G. (2014): Pollen morphology and its phylogenetic implications in the genus Picea. - Plant Systematics and Evolution 300: 461-473.

[14] Kormut'ák, A. (1996): Development and viability of silver fir pollen in air-polluted and non-polluted habitats in Slovakia. - Forest Genetics 3: 147-151.

[15] Krug, E. (1990): Reduced fertilization capacity of $\mathrm{SO}_{2}$-fumigated Picea omorika pollen. Forest Pathology 20: 122-126.

[16] Lin, J., Jach, M. E., Ceilemans, R. (2001): Stomatal density and needle anatomy of Scots pine (Pinus sylvestris) are affected by elevated $\mathrm{CO}_{2}$. - New Phytologist 150: 665-674.

[17] Matziris, D. I. (1984): Genetic variation in morphological and anatomical needle characteristics in the Black pine of Peloponnesos. - Silvae Genetica 33: 164-169.

[18] Meidner, H., Mansfield, T. A. (1968): Physiology of Stomata. - McGraw-Hill, London.

[19] Milovanović, J., Ivetić, V., Vilotić, D., Šijačić-Nikolić, M. (2005): Morpho-anatomical characteristics of needles from different Serbian spruce phenogroupes. - Acta Herbologica 14: 41-50.

[20] Nikolić, B., Bojović, S., Marin, P. D. (2015): Variability of morpho-anatomical characteristics of the needles of Picea omorika from natural populations in Serbia. - Plant Biosystems 149: 61-67.

[21] Nowak, D. J. (2002): The Effect of Urban Trees on Air Quality. - USDA Forest Service, Syracuse, NY.

[22] Pawlaczyk, E. M., Bobowicz, M. A. (2008): Silver fir (Abies alba Mill.) differentiation from Jata Reserve as expressed by morphological and anatomical features of needles. Leśne Prace Badawcze (Forest Research Papers) 69: 243-253.

[23] Pawlaczyk, E. M., Grzebyta, J., Bobowicz, M. A., Korczyk, A. F. (2005): Individual differentiation of Abies alba Mill, population from the Tisovik Reserve. Variability expressed in morphology and anatomy of needles. - Acta Biologica Cracoviencia, Series Botanica 47: 137-144.

[24] Peace, E. A., Lea, P. J., Darrall, N. M. (1995): The effect of open-air fumigation with $\mathrm{SO}_{2}$ and $\mathrm{O}_{3}$ on carbohydrate metabolism in Scots pine (Pinus sylvestris) and Norway spruce (Picea abies). - Plant, Cell \& Environment 18: 277-283. 
[25] Popović, T., Knežević, J., Jović, B., Marić-Tanasković, L., Dimić, B. (2016): Air Quality in Republic of Serbia in 2015. - Agency for Environmental Protection, Belgrade (in Serbian).

[26] Radovanović, B., Šinžar-Sekulić J, Rakić T, Lakušić, D. (2014): Variation in needle anatomy of Picea omorika (Pinaceae) plants belonging to different gene pools in natural populations on Tara Mt. In Serbia. - Botanica Serbica (Belgrade) 38: 237-246.

[27] Robakowski, P., Samardakiewicz, S., Kierzkowski, D. (2004): Variation in structure of needles of Silver Fir (Abies alba Mill.) saplings growing under the canopies of diverse tree species. - Polish Journal of Ecology 52: 563-568.

[28] Sancho-Knapik, D., Peguero-Pina, J. J., Flexas, J., Herbette, S., Cochard, H., Niinemets, Ü., Gil-Pelegrín, E. (2014): Coping with low light under high atmospheric dryness: shade acclimation in a Mediterranean conifer (Abies pinsapo Boiss.). - Tree Physiology 34: 1321-1333.

[29] Sękiewicz, K., Sękiewicz, M., Jasińska, A. K., Boratyńska, K., Iszkuło, G., Romo, A., Boratyński, A. (2013): Morphological diversity and structure of West Mediterranean Abies species. - Plant Biosystems 147: 125-134.

[30] Stefanović, M. (2015): Variability of terpene composition and morphological characteristics of Taxus baccata L. needles from Serbia (in Serbian). - Ph. D. Thesis, Faculty of Forestry, University of Belgrade.

[31] Qiang, W., Wang, X., Chen, T., Feng, H., An, L., He, Y., Wang, G. (2003): Variations of stomatal density and carbon isotope values of Picea crassifolia at different altitudes in the Qilian Mountains. - Trees - Structure and Function 17: 258-262.

[32] Vidaković, M. (1982): Četinjače. Morfologija i varijabilnost. - JAZU i Sveučilišna naklada Liber, Zagreb.

[33] Vukićević, E. (1987): Dekorativna dendrologija. - IŠRO Privredno finansijski vodič, Univerzitet u Beogradu, Beograd.

[34] Wahid, N., Gonzales-Martinez, S. C., Hadrami, I. E., Boulli, A. (2006): Variation of morphological traits in natural populations of maritime pine (Pinus pinaster Ait.) in Morocco. - Annals of Forest Sciences 63: 83-92.

[35] Wolf, L. (1950): Mikroskopicka tehnica. - Statni zdravotnicke nakladatelstva. Praha.

[36] Woodward, F. I., Bazzaz, J. D. (1988): The response of stomatal density to $\mathrm{CO}_{2}$ partial pressure. - Journal of Experimental Botany 39: 1771-1781. 